\title{
Transcription factor ETV1 is essential for rapid conduction in the heart
}

\author{
Akshay Shekhar, ${ }^{1}$ Xianming Lin, ${ }^{1}$ Fang-Yu Liu, ${ }^{1}$ Jie Zhang, ${ }^{1}$ Huan Mo, ${ }^{2}$ Lisa Bastarache, ${ }^{2}$ Joshua C. Denny, ${ }^{2}$ Nancy J. Cox, ${ }^{2}$ \\ Mario Delmar, ${ }^{1}$ Dan M. Roden, ${ }^{2}$ Glenn I. Fishman, ${ }^{1}$ and David S. Park ${ }^{1}$
}

${ }^{1}$ Leon H. Charney Division of Cardiology, New York University School of Medicine, New York, New York, USA. ${ }^{2}$ Vanderbilt University Medical Center, Nashville, Tennessee, USA.

\begin{abstract}
Rapid impulse propagation in the heart is a defining property of pectinated atrial myocardium (PAM) and the ventricular conduction system (VCS) and is essential for maintaining normal cardiac rhythm and optimal cardiac output. Conduction defects in these tissues produce a disproportionate burden of arrhythmic disease and are major predictors of mortality in heart failure patients. Despite the clinical importance, little is known about the gene regulatory network that dictates the fast conduction phenotype. Here, we have used signal transduction and transcriptional profiling screens to identify a genetic pathway that converges on the NRG1-responsive transcription factor ETV1 as a critical regulator of fast conduction physiology for PAM and VCS cardiomyocytes. Etv1 was highly expressed in murine PAM and VCS cardiomyocytes, where

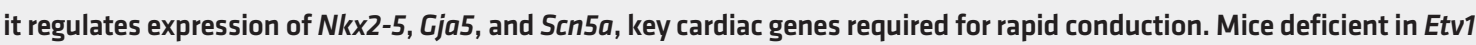
exhibited marked cardiac conduction defects coupled with developmental abnormalities of the VCS. Loss of Etv1 resulted in a complete disruption of the normal sodium current heterogeneity that exists between atrial, VCS, and ventricular myocytes. Lastly, a phenome-wide association study identified a link between ETV1 and bundle branch block and heart block in humans. Together, these results identify ETV1 as a critical factor in determining fast conduction physiology in the heart.
\end{abstract}

\section{Introduction}

Heritable and acquired syndromes affecting fast conduction in the atria and ventricular conduction system (VCS) produce a broad spectrum of arrhythmic disease, including atrial fibrillation, ventricular tachyarrhythmias, and heart block. In addition, aberrant VCS impulse propagation increases morbidity and mortality in heart failure patients because of electrical dyssynchrony (1). Despite the significant clinical impact of fast conduction disorders on the health care system, limited therapeutic options exist. Through an increased understanding of the molecular determinants of fast conduction, targeted therapies aimed at improving conduction parameters in the atria or VCS can be developed.

A hierarchy of myocardial conduction velocities exists within the heart to ensure optimal cardiac output. Fast conduction is an essential feature of the pectinated atrial myocardium (PAM) and VCS to coordinate and synchronize contraction of the cardiac chambers. The VCS is composed of Purkinje cells and is structurally divided into the His bundle, bundle branches, and the Purkinje fiber network (also collectively referred to as the HisPurkinje system). The specialized conduction properties of PAM and Purkinje myocytes are due to the enriched expression of key conduction genes, which include $\operatorname{Scn} 5 a$ (encoding the $\alpha$ subunit of the cardiac sodium channel $\mathrm{Na}_{\mathrm{v}} 1.5$ ) (2) and Gja5 (encoding the high-conductance gap junction protein connexin40, or $\mathrm{Cx} 40$ ) (3). This unique expression profile imparts to these cell types distinct

Conflict of interest: The authors have declared that no conflict of interest exists. Submitted: April 8, 2016; Accepted: September 15, 2016.

Reference information: J Clin Invest. 2016;126(12):4444-4459. doi:10.1172/JCI87968. electrophysiological features, including cell type-specific biophysical properties of the sodium current $(4,5)$ and rapid impulse propagation between neighboring cardiomyocytes $(6,7)$.

Common to all rapidly conducting tissues is their origins within the pectinated and trabeculated myocardium during atrial and ventricular chamber formation, respectively. During this stage, pectinated and trabecular myocytes grow as sheet-like layers along the endocardial surface of the cardiac chambers and acquire the fast conduction gene program, which is maintained into adulthood in the PAM and VCS. How these pectinated/trabecular myocytes acquire and maintain the fast conduction gene program is incompletely understood. Two transcription factors, the homeobox factor NKX2-5 and the T-box factor TBX5, are both known to work cooperatively in specification of the VCS (8-10). Mutations in NKX2-5 or TBX5 result in congenital heart disease and conduction defects, such as atrioventricular (AV) block and/ or bundle branch block in humans (11-15) and mouse models (10, 16-18). Yet what remains unresolved is how these factors, which are broadly expressed in the heart, contribute to the fast conduction phenotype only within pectinated and trabeculated myocytes that ultimately become the PAM and VCS, respectively. Moreover, a perinatal knockout of Nkx2-5 did not show an appreciable change in $\mathrm{Na}_{\mathrm{v}} 1.5$ expression in the atria and VCS (19). Therefore, we hypothesized that additional cell type-specific transcriptional regulators must be playing a role in activating the fast conduction gene program exclusively within the PAM and VCS.

To identify novel transcriptional regulators of the fast conduction phenotype, we made use of our previous observation that neuregulin-1 (NRG1) is sufficient to upregulate cardiac conduction system-lacZ (CCS-lacZ) reporter gene expression throughout 
A

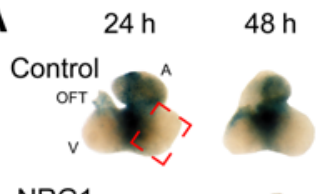

NRG1

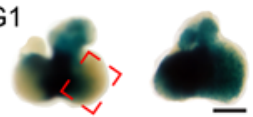

C

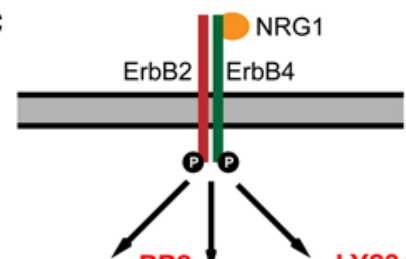

PP2

LY294002

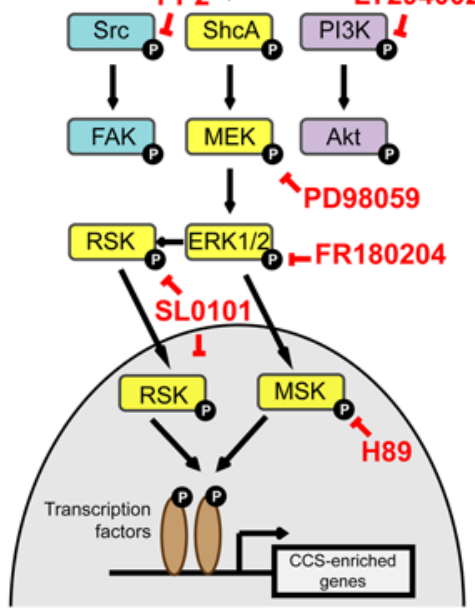

B

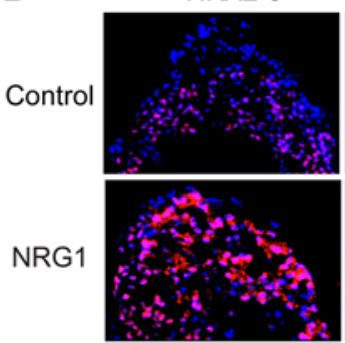

Cx40

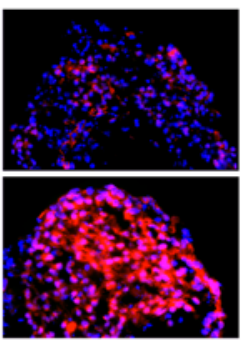

$\mathrm{Na}_{\mathrm{y}} 1.5$

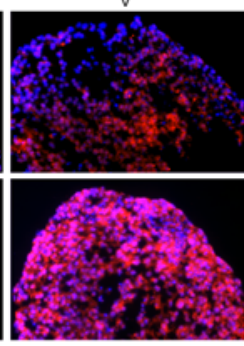

IRX3

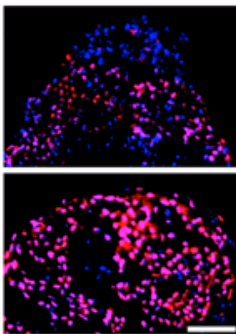

D

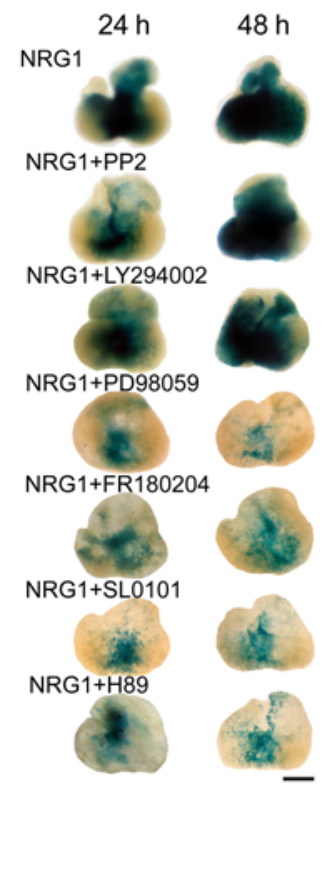

E

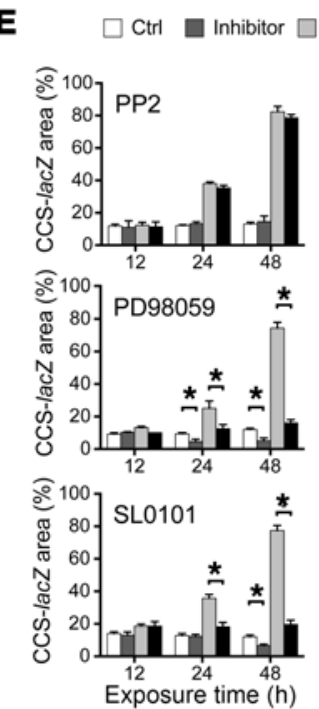

$\mathbf{F}$

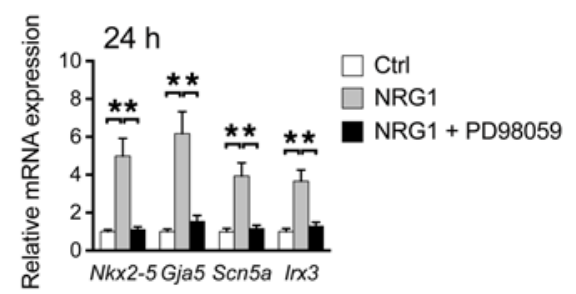

Figure 1. NRG1 regulates CCS-lacZ gene enrichment through MAPK signaling. (A) Representative E9.5 X-gal-stained CCS-lacZ hearts after in vitro culture with vehicle control or NRG1 for 24 or 48 hours. OFT, outflow tract; V, ventricle; A, atria. (B) Immunofluorescence staining of E9.5 hearts cultured with vehicle control or NRG1 for 24 hours for expression of NKX2-5, CX40, Na 1.5 , and IRX3. (C) Schematic representation of NRG1-ErbB2/ErbB4 intracellular signaling highlighting pathway-specific kinase inhibitors (red). (D) Representative E9.5 X-gal-stained CCS-lacZ hearts cultured with vehicle control or kinase inhibitors in the presence of NRG1. Top row: Representative images presented in A. (E) Quantification of CCS-lacZ expression, determined as a ratio of X-gal-positive area to the total heart area $(n=4)$. (F) Quantitative RT-PCR of Nkx2-5, Gja5, Scn5a, and Irx3 from E9.5 hearts in culture treated with vehicle control, NRG1, or NRG1 plus PD98059 for 24 hours. Red boxed region in A corresponds to location of immunofluorescence image in B. Nuclei were stained with DAPI (blue, B). NRG1 concentration for all experiments was $2.5 \times 10^{-9} \mathrm{M}$. Doses used for kinase inhibitor studies were as follows: PP2 (10 $\mu \mathrm{M}$ ), LY294002 $(25 \mu \mathrm{M})$, PD98059 $(50 \mu \mathrm{M})$, FR180204 $(20 \mu \mathrm{M})$, SL0101 $(50 \mu \mathrm{M})$, H89 $(10 \mu \mathrm{M})$. Scale bars: $200 \mu \mathrm{m}(\mathbf{A}$ and D); $50 \mu \mathrm{m}(\mathbf{B})$. Data represent mean \pm SEM. ${ }^{*} P<0.05,1$-way ANOVA.

embryonic hearts in culture $(20,21)$. Based on this observation, we performed a 2-component screen using a signal transduction inhibitor assay in CCS-lacZ hearts in tandem with gene profiling of developing and mature Purkinje cells. Through this dual screen, a single candidate, Etv1, a member of the E-twenty-six (ETS) transcription factor family, was identified. ETV1 is a NRG1-responsive factor that is highly expressed in PAM and VCS myocytes, where it establishes the fast conduction phenotype through enrichment of Nkx2-5, Gja5, and Scn5a. Consequently, Etv1-deficient mice exhibit cardiac conduction defects and developmental abnormalities of the VCS. Etv1 KO hearts display a loss of the normal sodium current heterogeneity that exists between Purkinje, atrial, and ventricular myocytes. A phenome-wide association study (PheWAS) (22) identified associations between ETV1 and bundle branch block and heart block in humans, suggesting conservation of this ETV1-dependent pathway in mice and humans.

\section{Results}

NRG1 upregulates CCS-lacZ reporter gene expression in embryonic hearts through an RSK/MSK-dependent signaling pathway. We previously showed that NRG1 treatment of embryonic day 9.5 (E9.5) CCS-lacZ hearts in culture was able to significantly increase 

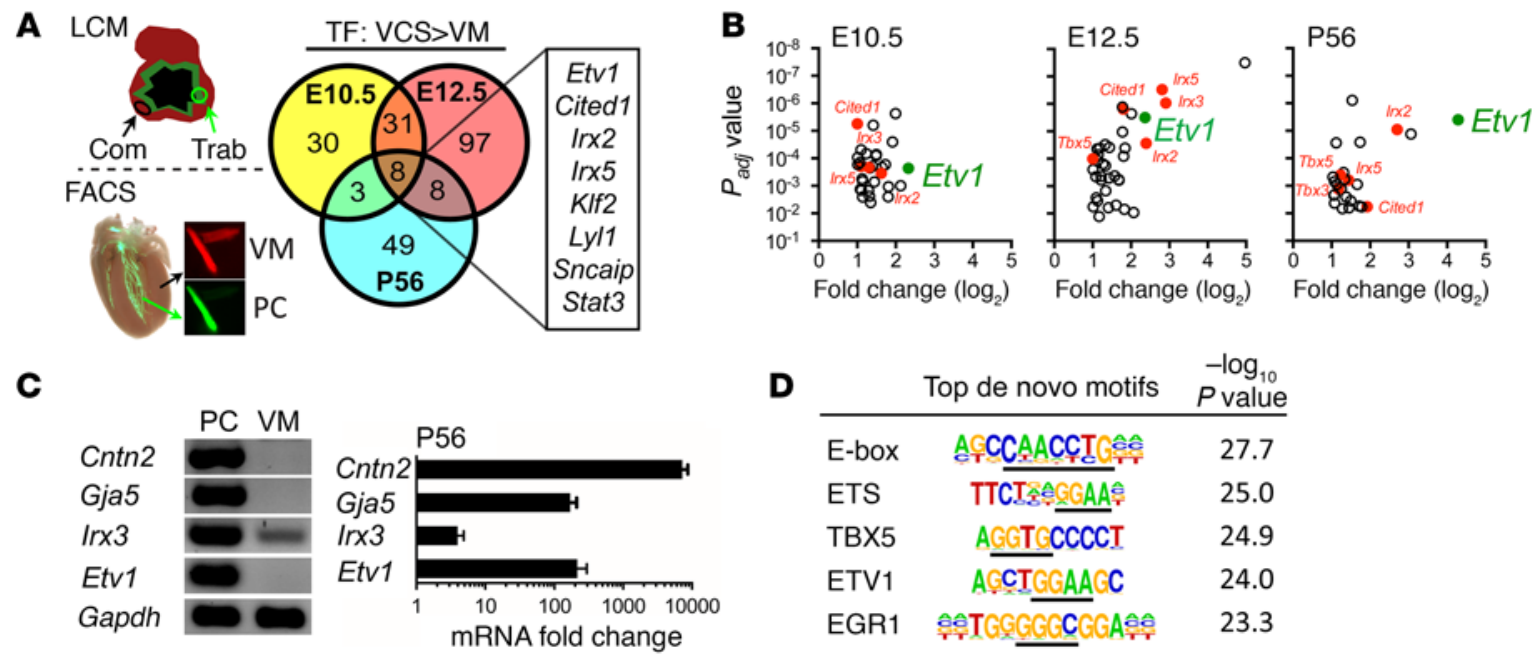

\begin{tabular}{|c|c|c|}
\hline & Top de novo motifs & $\begin{array}{c}-\log _{10} \\
P \text { value }\end{array}$ \\
\hline E-box & ACCCAACCTG & 27.7 \\
\hline ETS & TTCT 중스GAA & 25. \\
\hline TBX5 & AGGT CCCCT & 24.9 \\
\hline ETV1 & ACCTGGAACC & 24.0 \\
\hline EGR1 & 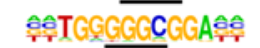 & 23. \\
\hline
\end{tabular}

Figure 2. NRG1-MAPK target gene Etv1 is highly expressed in developing and mature VCS cells. (A) Gene expression profiling of laser capture microdissected (LCM) trabecular myocytes (Trab) versus compact zone myocytes (Com) (E10.5, E12.5) $(n=2)$ and FACS-sorted Purkinje cells (PC) versus ventricular myocytes (VM) (P56; $n=4)$. Venn diagram of VCS transcription factors (TF) from 3 data sets reveals 8 genes significantly enriched at all time points.

(B) Scatter plots identified Etv1 (green) as one of the most enriched transcription factors in developing and mature VCS cells. (C) Semiquantitative and quantitative RT-PCR analysis confirmed significant Etv1 enrichment in adult Purkinje cells in addition to other known markers of the VCS (Cntn2, Cja5, Irx3) $(n=3)$. (D) Top consensus sequence motifs enriched in promoters $1 \mathrm{~kb}$ upstream of the known transcription start site in Purkinje-enriched genes. VCS, ventricular conduction system. Data represent mean \pm SEM.

reporter gene expression (20). To confirm that NRG1 treatment upregulates bona fide fast conduction genes and not just CCSlacZ expression (Figure 1A), we immunostained treated hearts for NKX2-5, Cx40, $\mathrm{Na}_{\mathrm{v}} 1.5$, and Iroquois-related homeobox 3 (IRX3) (ref. 23 and Figure 1B). NRG1 treatment enriched expression of these gene products throughout the heart. Next, we sought to identify the critical NRG1-dependent signal transduction pathway that mediates CCS-lacZ and fast conduction gene enrichment using a kinase inhibitor strategy.

Binding of NRG1 to its cognate receptor, ErbB4, results in heterodimerization with ErbB2, which activates numerous downstream signal transduction events, including Src, PI3K, and the Ras-MAPK pathways (refs. 24-27; and schematic, Figure 1C). Selective inhibition of Src or PI3K signaling did not perturb NRG1-dependent CCS-lacZ upregulation (Figure 1, D and E). In contrast, inhibition of MEK in the Ras-MAPK pathway completely abrogated CCS-lacZ enrichment in response to NRG1 treatment (Figure 1, D and E). MEK inhibition also reduced basal levels of CCS-lacZ expression (Figure 1E and Supplemental Figure 1; supplemental material available online with this article; doi:10.1172/ JCI87968DS1). Inhibition of downstream targets of MEK, which include ERK1 and ERK2, 90-kDa ribosomal S6 kinase (RSK), and mitogen- and stress-activated protein kinase (MSK), resulted in similar blockade of NRG1-mediated CCS-lacZ upregulation (Figure 1, D and E, and ref. 28). Confirming the CCS-lacZ reporter screen, NRG1 treatment of E9.5 hearts significantly increased transcript levels of Nkx2-5, Gja5, Scn5a, and Irx3 (Figure 1F), while cotreatment with NRG1 plus MEK inhibitor (PD98059) completely blocked fast conduction gene upregulation.

The NRG1- and MAPK-responsive transcription factor Etv1 is highly enriched in fast conduction tissues. In parallel with the signal transduction screen, we generated differential gene expres- sion libraries at multiple developmental time points (E10.5, E12.5, and postnatal day 56 [P56]; ref. 29) to identify Purkinjeenriched transcription factors (Figure $2 \mathrm{~A}$ ). For embryonic stages, trabecular myocytes, from which VCS cells derive, were separated from compact ventricular myocytes using laser capture microdissection. For adult stages, we used a dual reporter system to identify cardiomyocytes (Myh6-Cre LSL-tdTomato) and cardiac conduction system cells $\left(C_{\left.n t n 2^{E G F P /+}\right)}(29,30)\right.$. Myh6-Cre LSL-tdTomato Cntn2 $2^{\text {EGFP/+ }}$ hearts were enzymatically dissociated to collect ventricular myocytes (identified as Tomato $^{+} /$ CNTN2 ${ }^{\text {EGFP-) }}$ and Purkinje cells (identified as Tomato $^{+} / \mathrm{CNT}$ $\mathrm{N} 2^{\mathrm{EGFP}}$ ) by flow cytometry (29). Expression libraries were then generated using the Affymetrix gene array platform. Analysis of our gene lists for VCS-enriched transcription factors yielded 8 candidate factors present at all developmental time points (Figure 2A). Etv1 was the most highly enriched transcription factor in adult Purkinje cells (Figure 2B). Etv1 enrichment in Purkinje cells was confirmed by quantitative RT-PCR (qPCR) (Figure 2C). Interestingly, ETV1 is a known target of ErbB2 (31-34) and Ras-MAPK-RSK/MSK signaling pathways (34-36) and was therefore an attractive candidate.

Etv1 belongs to the PEA3 group of ETS family transcription factors, which are involved in cell fate decisions and in functional modulation of neuronal cell types (37-39). ETS family members regulate transcription through the ETS-DNA binding domain that recognizes a central 5'-GGAA/T-3' motif (40). Using an unbiased approach, we performed transcription factor motif analysis on the promoters of the most highly enriched genes in the Purkinje data set, restricting our analysis to $1,000 \mathrm{bp}$ upstream of the transcriptional start sites. The generalized ETS-binding motif (- $\log P=25.0)$ and the ETV1-binding motif ( $\log P=24.0$ ) were the second and fourth most significantly enriched motifs in the Purkinje-enriched gene set (Figure 2D). 
A $\quad \mathrm{E} 12.5$

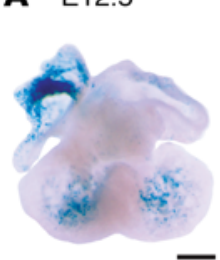

E16.5

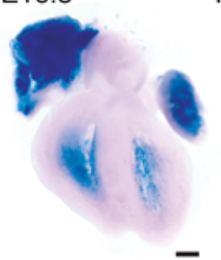

B

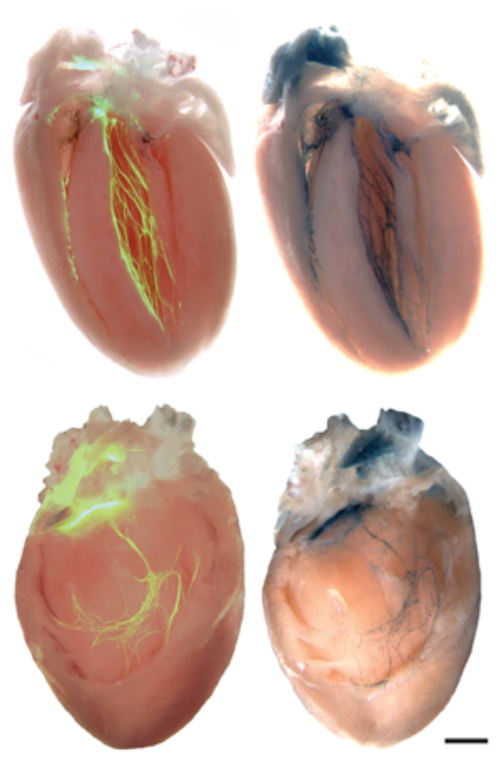

P21

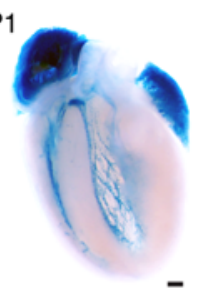

P70

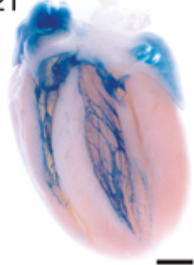

C
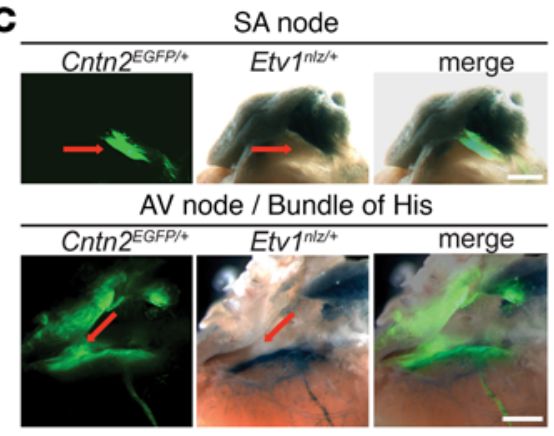

Purkinje cells
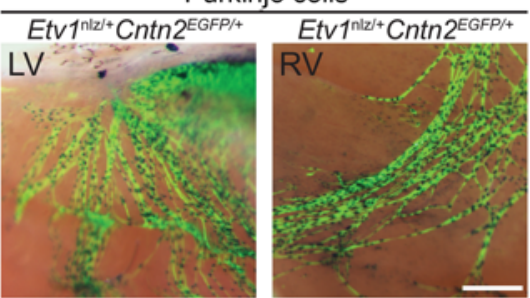

Figure 3. ETV1 expression is enriched in fast conduction tissues of embryonic and adult mammalian hearts. (A) Whole-mount X-gal staining of $E t v 1^{n / 2 /+}$ murine hearts demonstrated Etv1 expression in regions of fast conduction (atrial myocytes, His-Purkinje network) within developing and mature hearts (E12.5, E16.5, P1, P21, P70). (B) Low-magnification views of lacZ expression within $E t v 7^{\text {nlz/+ }}$ Cntn2 ${ }^{E G F P /+} \mathrm{P} 21$ hearts revealed identical expression of Cntn2-EGFP and Etv1-nlz in the left and right VCS. (C) Higher magnification of the Purkinje cell network showed overlapping expression of Cntn2-EGFP and Etv1-n/z within all VCS cells (bottom panels). Etv1-nlz expression was significantly lower in slowly conducting CNTN2$\mathrm{EGFP}^{+} \mathrm{SA}$ nodal (top panels) and AV nodal (middle panels) cells. Red arrows identify CNTN2-EGFP ${ }^{+}$ nodal regions. (D) Immunofluorescence staining for ETV1 in Cntn2 $2^{E C F P /+}$ murine heart sections. ETV protein is expressed in Cx40-positive atrial myocytes and colocalizes with CNTN2-EGFP ${ }^{+}$VCS cells with robust nuclear expression (white arrows). (E and F) Cardiac ETV1 expression pattern was conserved in pigs and humans. In Yucatan miniature pig ( 2 years old), ETV1 colocalizes with $C x 40$ in atrial myocytes and VCS cells $(\mathbf{E})$, and in human fetal heart (16 weeks), ETV1 colocalizes with $C \times 40$ in the atria and CNTN2 in Purkinje cells (F). SA, sinoatrial; AV, atrioventricular; PAM, pectinated atrial myocardium; His, bundle of His; PC, Purkinje cells. Scale bars: $100 \mu \mathrm{m}$ (E12.5, E16.5, P1), $1 \mathrm{~mm}$ (P21, P70) (A); $1 \mathrm{~mm}$ (B); $500 \mu \mathrm{m}$ (C); $20 \mu \mathrm{m}$ (D-F).
D

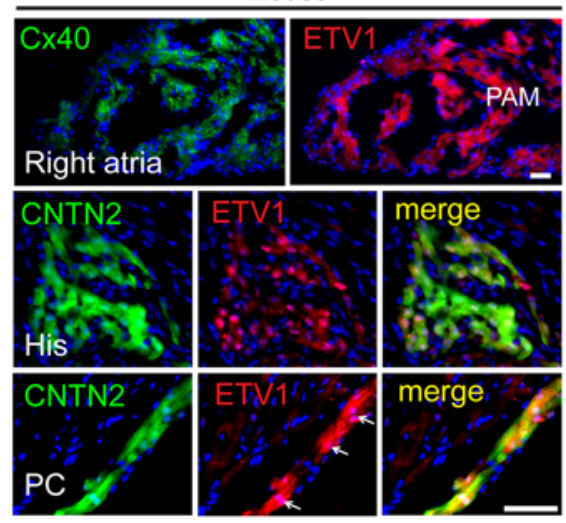

E

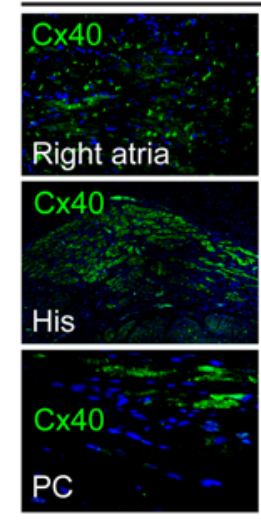

Pig

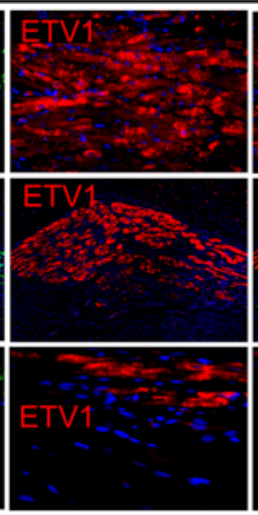

$\mathbf{F}$

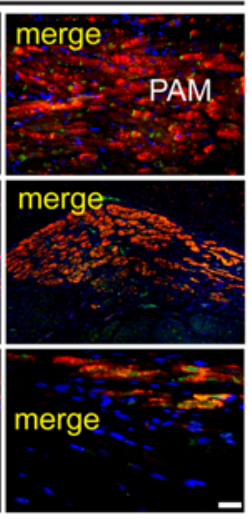

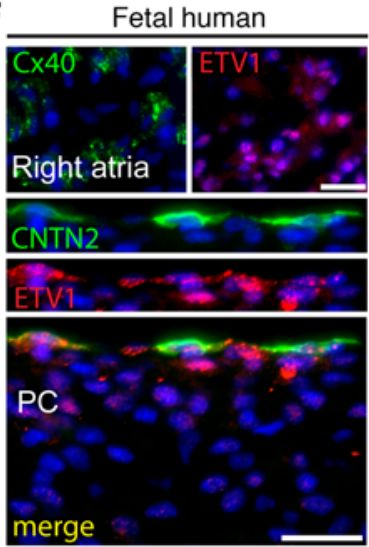

We next examined the temporal and regional expression of Etv1 during heart development using Etv1 ${ }^{n l z /+}$ knockin reporter mice, which express a lacZ reporter gene with a nuclear localization signal (38). Cardiac expression of Etv1 was highly abundant in all fast conduction tissues throughout development (Figure 3A). At embryonic stages, Etv1 was localized to the PAM and trabecular myocardium of the ventricles. At postnatal time points, Etv1 expression remained in the PAM but was predominantly restricted to the VCS in the ventricles (Figure 3A and Supplemental Figure 2). Etv1 was highly abundant in the His-Purkinje network, as visualized by the Cntn $2^{E G F P /+}$ reporter (Figure 3, B and C, bottom panels). Histological evaluation of Etv1 ${ }^{l z /+} \mathrm{P} 1$ hearts showed high levels of reporter gene expression in the PAM (Supplemental Figure
2, B and C) and the His-Purkinje system (Supplemental Figure 2, $\mathrm{D}$ and E). Etv1 was expressed at lower levels in the sinoatrial and AV nodes (Figure 3C, top and middle panels, red arrows). Immunofluorescence staining for ETV1 recapitulated $E t v{ }^{1 l z /+}$ reporter gene expression with high levels detected in the PAM and VCS in adult mouse hearts (Figure 3D). The cardiac expression pattern of ETV1 was conserved in mature pig (Figure 3E) and embryonic human hearts (Figure 3F).

Etv1 expression is NRG1 responsive in embryonic and postnatal hearts. As NRG1 treatment can expand CCS-lacZ expression transmurally in embryonic hearts, we tested whether NRG1 would have a similar effect on Etv1 expression. Treatment of E9.5 Etv ${ }^{l l z /+}$ hearts with NRG1 expanded reporter gene expression from an 
A

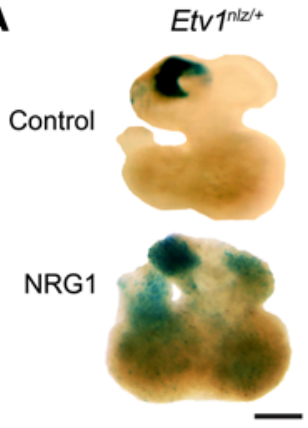

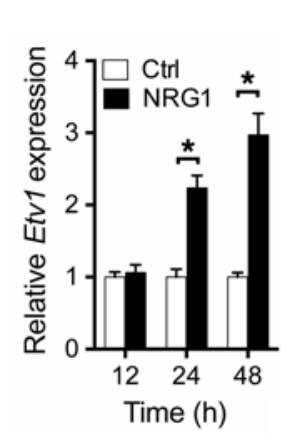

ETV1

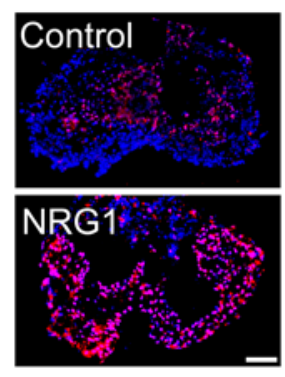

B

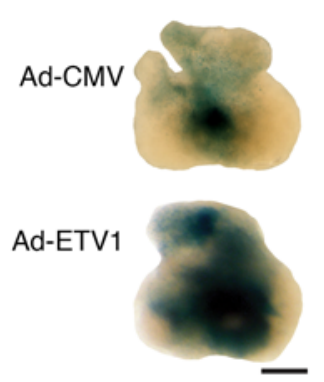

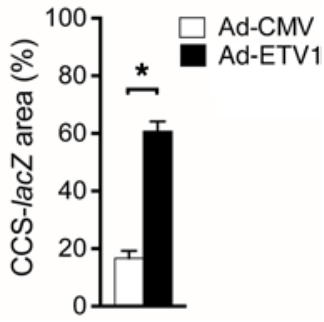

C

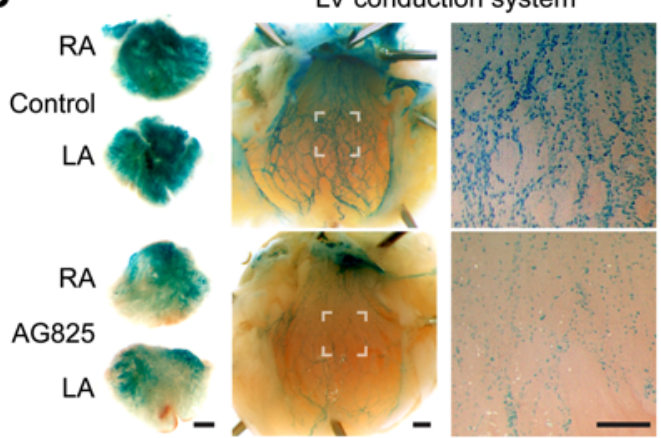

D

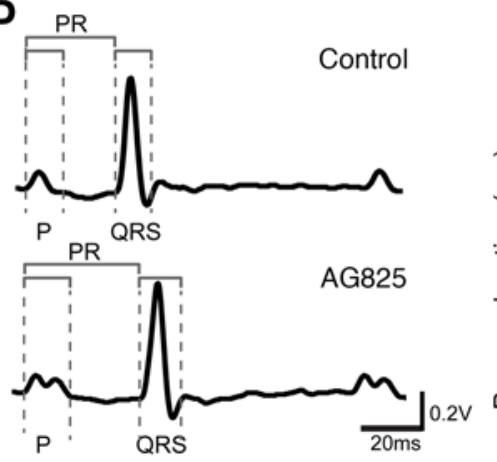

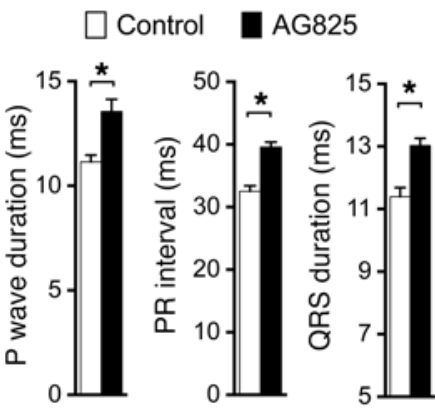

Figure 4. ETV1 is regulated by NRG1 signaling and is sufficient to drive CCS-lacZ expression. (A) Representative E9.5 X-gal-stained Etv1 $7^{n / 2 /+}$ hearts after in vitro culture with vehicle control or NRG1 for 24 hours (left panels). Quantitative RT-PCR for Etv1 in E9.5 hearts cultured with vehicle control or NRG1 for 12 , 24, or 48 hours ( $n=3$, middle panel). Immunofluorescence staining for ETV1 in E9.5 hearts cultured with vehicle control or NRG1 for 24 hours (right panels). (B) E9.5 X-gal-stained CCS-lacZ hearts after in vitro culture with Ad-CMV or Ad-ETV1 for 48 hours. Quantification of CCS-lacZ expression, determined as a ratio of lacZ-positive area to the total heart area $(n=3)$. (C) P1 Etv $7^{n / 2 /+}$ mice were treated with AC825 $(1 \mathrm{mg} / \mathrm{kg}$, i.p.) daily for 7 days. Representative examples of X-gal-stained P8 Etv1 $1^{n / 2 /+}$ hearts showed significant reduction of Etv1-n/z reporter gene expression with AC825 treatment $(n=8)$. White boxed region in middle panels corresponds to the location of higher-magnification images in the right panels. (D) Representative surface ECC traces of P8 Etv ${ }^{1 / 2 /+}$ mice treated with vehicle control or AC825 for 7 days $(n=8)$. LV, left ventricle; RA, right atria; LA, left atria. Data represent mean \pm SEM. ${ }^{*} P<0.05$, 2 -tailed Student's $t$ test. Scale bars: $50 \mu \mathrm{m}$ (A and B); $100 \mu \mathrm{m}$ (C).

exclusively trabecular pattern to a transmural distribution (Figure 4A, left). NRG1 treatment increased Etv1 RNA levels at 24 and 48 hours (Figure 4A, middle). Immunostaining of NRG1-treated hearts showed a similar expansion of ETV1 throughout the heart by 24 hours (Figure 4A, right). We next examined whether ETV1 is sufficient to increase the CCS-lacZ reporter independent of exogenous NRG1. Cultured E9.5 CCS-lacZ hearts were exposed to Adenovirus-ETV1 (Ad-ETV1) or Ad-CMV control. Ad-ETV1treated hearts displayed enhanced CCS-lacZ expression, whereas Ad-CMV control had no effect (Figure 4B). To evaluate whether NRG1 signaling is required to maintain Etv1 expression in postnatal hearts, Etv1 ${ }^{n z /+}$ P1 mice were treated daily for 7 days with AG825 (41, 42), an inhibitor of ErbB2. AG825 treatment caused downregulation of ETV1 ${ }^{\text {nlz }}$ reporter gene expression in the PAM and Purkinje cells (Figure 4C). Importantly, mice treated with AG825 also exhibited conduction abnormalities on ECG, displaying significantly prolonged $\mathrm{P}$ wave, PR interval, and QRS wave durations (Figure 4D).

Cardiac ETV1 is posttranslationally regulated by NRG1 signaling. The Ras-MAPK-RSK/MSK signaling cascade has been shown to positively regulate the transcriptional activity of ETV1 through phosphorylation of key serine residues in heterologous expression systems $(35,36)$. To evaluate whether this signaling axis is operative in the heart, we first stained E13.5 (Figure 5A) and P21 hearts
(Figure 5B) for activated components of the NRG1-ErbB2/4MAPK signaling cascade. At E13.5, NRG1 expression was restricted to endocardial cells that were in direct contact with pectinated and trabecular myocytes. Phosphorylated (activated) forms of ErbB4 (pErbB4), ErbB2 (pErbB2), and ERK1/2 (pERK1/2) were restricted to PAM and ventricular trabecular myocytes, mirroring the expression patterns of ETV1 and Cx40 in serial sections (Figure 5A). At P21, $\mathrm{NRG}^{+}$(red) cells are interdigitated among PAM and $\mathrm{CNTN2}^{+}$(green) Purkinje cells (Figure 5B). In the proximal and distal VCS, NRG1 ${ }^{+}$cells can be seen making numerous connections with CNTN2 ${ }^{+}$Purkinje cells (Figure 5B). Correspondingly, the PAM and CNTN2 ${ }^{+}$Purkinje cells are highly enriched in activated pErbB4 (Figure 5C). Phosphorylated (activated) forms of ERK1/2 and RSK were also enriched in PAM and Purkinje cells in P21 hearts (Supplemental Figure 3). To test dynamics of ETV1 activation, we treated dissociated neonatal rat atrial cardiomyocytes with NRG1, which resulted in serine phosphorylation (Figure 5D) and nuclear accumulation (Figure 5E) of ETV1.

Mice deficient in Etv1 exhibit cardiac conduction defects. To investigate whether ETV1 is an important regulator of cardiac conduction physiology, we performed electrophysiological analysis on $E t v 1^{n l z / n l z}$ mice, herein referred to as Etv1 knockout (KO) mice. As Etv1 KO mice die uniformly at 3 weeks of age due to severe neuromuscular impairment, ECG analysis was per- 
A
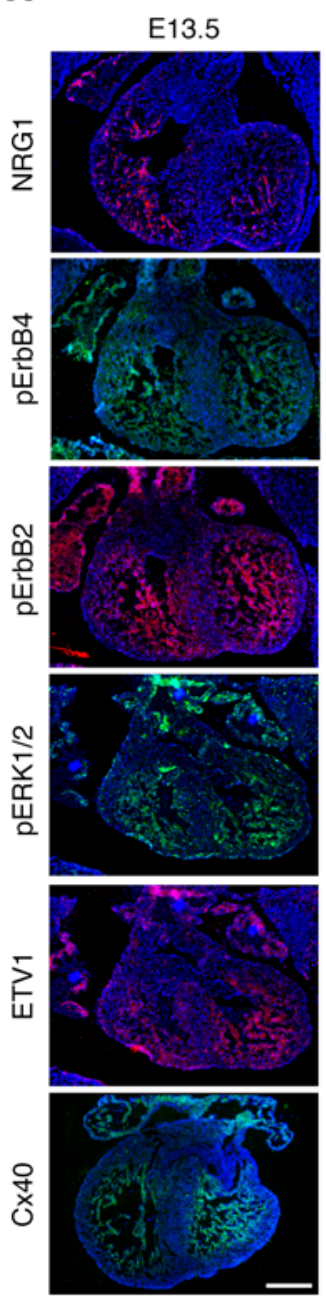

B

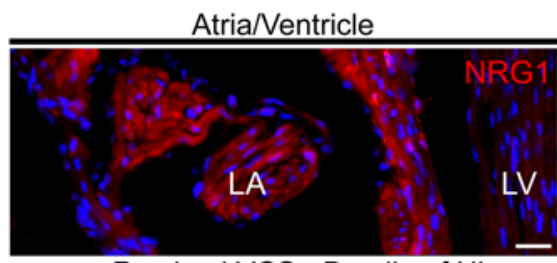

Proximal VCS - Bundle of His

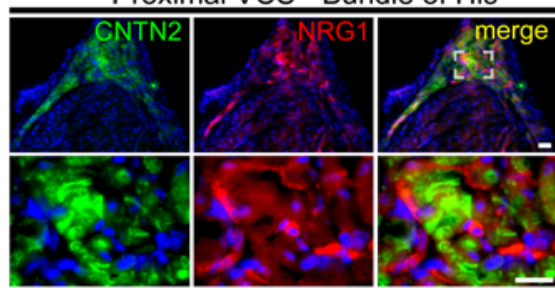

Distal VCS - Purkinje cells
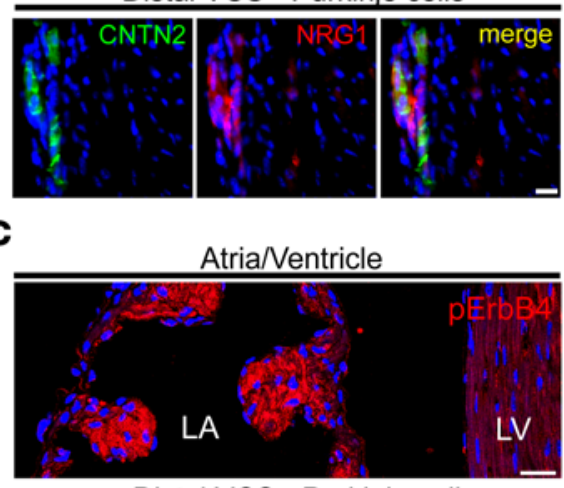

Distal VCS - Purkinje cells

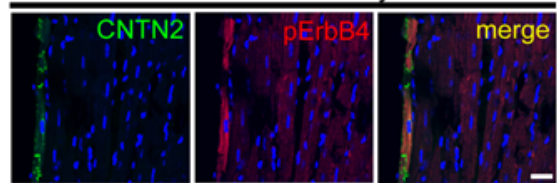

D IP: ETV1
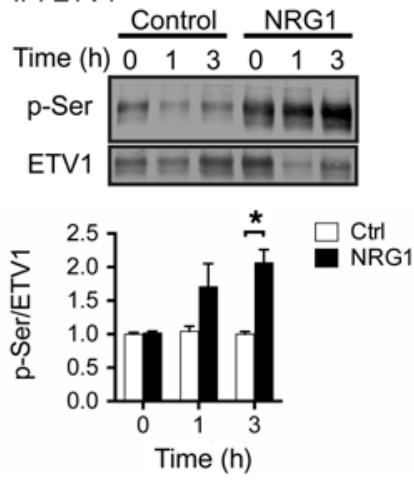

$\mathbf{E}$
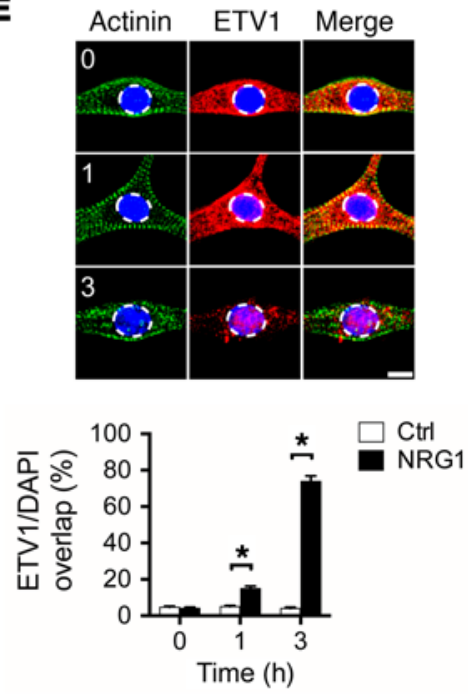

Figure 5. Activated NRG1 signaling is restricted to regions of fast conduction and results in activation of ETV1. (A) Immunofluorescence staining of NRG1, pErbB4, pErbB2, pERK1/2, ETV1, and Cx40 in E13.5 embryonic heart serial sections. (B and C) Immunofluorescence staining of NRG1 (B) and pErbB4 (C) in P21 atria and the His-Purkinje system. (D and E) Cultured neonatal rat atrial myocytes treated with vehicle control or NRG1 for 0, 1, or 3 hours were evaluated for ETV1 phosphorylation and nuclear accumulation. (D) ETV1 was immunoprecipitated from cell lysates followed by Western blot analysis to detect phosphoserine residues (p-Ser). Densitometry quantification of p-Ser levels (normalized to ETV1) presented relative to baseline $(n=4)$. (E) Nuclear accumulation of ETV1 with NRG1 treatment was detected using immunofluorescence staining. Percentage of ETV1 nuclear-positive area presented with respect to DAPIpositive area (dashed circles) ( $n=20$ cells). Nuclei were stained with DAPI; atrial myocytes were identified by $\alpha$-actinin staining (blue, DAPI; red, ETV1; green, actinin). LA, left atria; LV, left ventricle. Data represent mean \pm SEM. ${ }^{*} P<0.05$, 2-tailed Student's $t$ test. Scale bars: $50 \mu \mathrm{m}$ (A); $25 \mu \mathrm{m}$ (B and C); $5 \mu \mathrm{m}$ (E).

formed at P18 (38). At this age, Etv1 KO mice were smaller than their WT and heterozygous (Het) littermates but appeared relatively normal except for the previously described neuromuscular phenotype (38). Like AG825-treated mice, Etv1-deficient mice displayed cardiac conduction abnormalities as demonstrated by lengthening of the P wave, PR interval, and QRS wave durations (Figure 6A and Supplemental Table 1). In addition, $30 \%$ of Etv1 KO mice displayed bundle branch block (Figure 6A). Bundle branch blocks were never observed in WT or Het animals. Prolongation of the PR interval prompted further analysis of AV conduction using intracardiac electrogram recordings, which demonstrated prolonged atrial-His (AH) and His-ventricular (HV) intervals in Etv1 KO mice (Figure 6B and Supplemental Table 1). The $\mathrm{AH}$ interval is a surrogate measure of $\mathrm{AV}$ nodal conduction time, and the HV interval is a measure of VCS-dependent ventric- ular activation time. Etv1 $\mathrm{KO}$ mice had normal cardiac function as assessed by transthoracic echocardiography (Supplemental Table 2). Although Etv1 KO hearts were significantly smaller than their WT and Het counterparts, the heart weight-to-body weight ratios remained equivalent to those of control animals (Supplemental Figure 4A). There was no evidence of structural abnormalities or fibrosis in Etv1 KO hearts based on H\&E (Supplemental Figure $4 \mathrm{~B}$ ) or trichrome staining (Supplemental Figure 4, C and D). In addition, Etv1 KO hearts did not display abnormalities of cell cycling (Supplemental Figure 5) or increased apoptosis by TUNEL staining (data not shown).

Etv1-deficient mice have reduced levels of Nkx2-5, Gja5, and Scn5a in the atria and VCS. Conduction abnormalities in Etv1 KO mice were evaluated using immunofluorescence staining to detect altered expression of fast conduction genes. The normal 
A

Etv1 WT

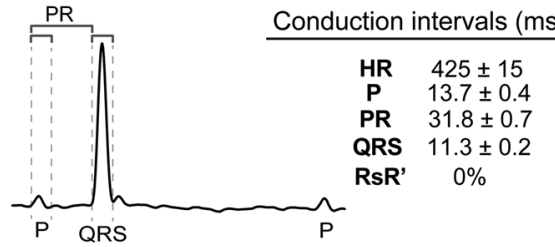

Etv1 KO

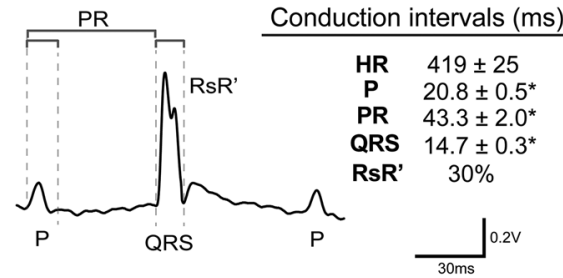

B

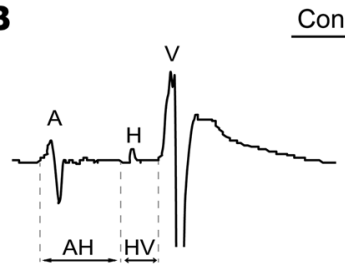

Conduction intervals (ms)

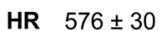

AH $20.3 \pm 0.9$

HV $7.2 \pm 0.4$

Conduction intervals (ms)

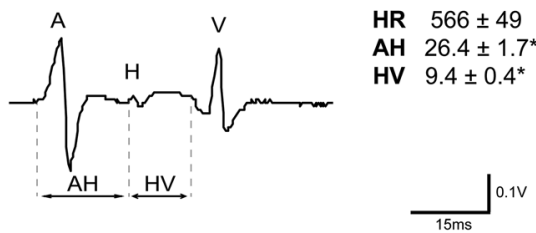

Figure 6. Etv1 mutant mice display conduction slowing. (A) Representative surface ECG traces of P18 Etv1 WT and KO mice. Conduction intervals from Etv1 KO mice showed significantly prolonged P, PR, and QRS intervals in comparison with WT mice $(n=10)$. A subset of Etv1 KO mice (30\%) displayed an RsR' pattern. (B) Intracardiac recordings in Etv1 KO mice showed significantly prolonged AH, HV, and AVI intervals in comparison with WT mice $(n>5)$. HR, heart rate; $\mathrm{AH}$, atrial-His; $\mathrm{HV}$, His-ventricular. Data represent mean $\pm \mathrm{SEM} .{ }^{*} P<0.05$, 2-tailed Student's $t$ test.

enrichment of $\mathrm{NKX} 2-5, \mathrm{Cx} 40$, and $\mathrm{Na}_{\mathrm{v}} 1.5$ in the atria, proximal VCS, and distal Purkinje cells was disrupted in P18 Etv1 KO hearts (Figure 7, A-C, and Supplemental Figure 6). NKX2-5 expression and $\mathrm{Na}_{\mathrm{v}} 1.5$ expression in the atria and VCS were reduced to ventricular levels. Similar reductions in NKX2-5 and $\mathrm{Na}_{\mathrm{v}} 1.5$ expression levels in atria and ventricular trabecular myocytes were evident in E13.5 Etv1 KO hearts (Supplemental Figure 7). In contrast, IRX3 levels remained unchanged in Etv1-deficient P18 hearts (Supplemental Figure 8). Western blot analysis of atrial samples from Etv1 KO mice demonstrated significantly reduced levels of NKX2-5, Cx40, and $\mathrm{Na}_{\mathrm{v}} 1.5$ compared with WT and Het controls (NKX2-5, 55\% $\pm 5 \%$; Cx40, 50\% $\pm 8 \%$; and $\mathrm{Na}_{\mathrm{v}} 1.5,64 \% \pm 7 \% ; P$ $<0.05$; KO vs. WT) (Figure 7, D and E, and Supplemental Table 3). To detect changes in Nkx2-5, Gja5, Scn5a, and Tbx5 transcript levels, we performed qPCR on FACS-purified atrial, Purkinje, and ventricular myocytes dissociated from Etv1 WT and KO mice in a Cntn2 $2^{E G F P /+}$ background. We used the mitochondrial red dye tetramethylrhodamine methyl ester perchlorate (TMRM) to isolate the cardiomyocyte fraction (43). Purkinje cells were sorted based on TMRM $^{\text {hi }}$ CNTN2 $^{\text {EGFP+ }}$ status. Nkx2-5, Gja5, and Scn5a transcript levels were significantly reduced in Etv1 KO atrial and Purkinje myocytes (Figure $7 \mathrm{~F}$ and Supplemental Table 4). Similar to the immunostaining results, $N k \times 2-5$ and $S c n 5 a$ RNA levels from Etv1 KO atrial and Purkinje myocytes were reduced to ventricular levels (Supplemental Figure 9). Interestingly, Tbx5 expression was significantly increased in mutant atrial and Purkinje myocytes, presumably through a compensatory mechanism (Figure 7F and Supplemental Table 4). We next treated E9.5 Etv1 WT and KO hearts in culture with NRG1 and measured transcript levels of fast conduction genes. With NRG1 treatment, Etv1 KO hearts had a significantly blunted response of Nkx2-5, Gja5, and Scn5a RNA upregulation compared with WT (Figure 7G).

Etv1-deficient mice exhibit hypoplasia of the VCS due to a reduction in terminal Purkinje cells. Given the reduced levels of NKX25 in the VCS of Etv1 KO hearts, a detailed structural evaluation of the His-Purkinje system was performed. In humans, NKX2-5 mutations cause nonsyndromic congenital heart defects and AV conduction abnormalities (14). Mouse models of Nkx2-5 haplo- insufficiency phenocopy the human condition and manifest prolonged PR and QRS durations on ECG as well as hypoplasia of the VCS with approximately $50 \%$ loss of terminal Purkinje cells $(17,18,44,45)$. To identify morphological defects in the conduction system, Etv1 KO mice backcrossed into the Cntn $2^{\text {EGFP/+ }}$ background were studied. The left and right VCS of Etv1 KO hearts was hypomorphic, recapitulating the defects seen in Nkx2-5 haploinsufficient mice (Figure 8A, left and middle panels, and Supplemental Figure 10). Regional analysis of the left VCS (Figure 8B) demonstrated significant loss of terminal Purkinje cells, while the fascicles appeared less affected. X-gal staining of Etv1 ${ }^{n l z / n l z}$ mice confirmed the absence of terminal Purkinje cells (Figure 8A, right panels). To quantify the percentage of CNTN2 $2^{\text {EGFP+ }}$ Purkinje cells in WT and KO hearts, ventricles were dissociated into single cells and stained with TMRM to identify cardiomyocytes (43). The percentage of $\mathrm{TMRM}^{\mathrm{hi}} \mathrm{CNTN}^{\mathrm{EGFP+}}$ Purkinje cells relative to total ventricular myocytes was reduced by $52 \%$ in Etv1 KO hearts as assessed by flow cytometry (Figure $8 \mathrm{C}$ ).

Etv1 KO hearts display loss of sodium current heterogeneity between Purkinje, atrial, and ventricular myocytes. Based on the conduction abnormalities in Etv1 KO mice and the reductions in Scn5a expression in atrial and Purkinje cells, we next studied the biophysical properties of the sodium current $\left(\mathrm{I}_{\mathrm{Na}}\right)$ in dissociated myocytes. The fast, voltage-gated $\mathrm{I}_{\mathrm{Na}}$ is a major determinant of myocardial excitability and conduction velocity. The biophysical properties of the $\mathrm{I}_{\mathrm{Na}}$ are not homogeneous between the atrial, Purkinje, and ventricular myocytes $(4,5)$. Atrial (4) and Purkinje (5) myocytes have greater maximum sodium conductance and correspondingly higher levels of $\mathrm{Na}_{\mathrm{v}} 1.5$ expression $(2,19)$. To verify and explore in greater detail the baseline $\mathrm{I}_{\mathrm{Na}}$ heterogeneity that exists between these major cardiac cell types, we applied whole-cell patch clamp technique to myocytes dissociated from $C n t n 2^{E G F P /+}$ P18 hearts. The maximum conductance of $\mathrm{I}_{\mathrm{Na}}$ was significantly different between all 3 cell types, with Purkinje (CNTN2 ${ }^{\mathrm{EGFP}+}$ ) cells having the greatest maximum conductance followed by atrial then ventricular myocytes $(1.11 \pm 0.09 \mathrm{nS} / \mathrm{pF}, 0.78 \pm 0.03 \mathrm{nS} / \mathrm{pF}$, $0.61 \pm 0.03 \mathrm{nS} / \mathrm{pF}$, respectively, $P<0.05$ ) (Figure $9 \mathrm{~A}$ and Supplemental Tables 5 and 6). Half-activation and inactivation voltages 
A
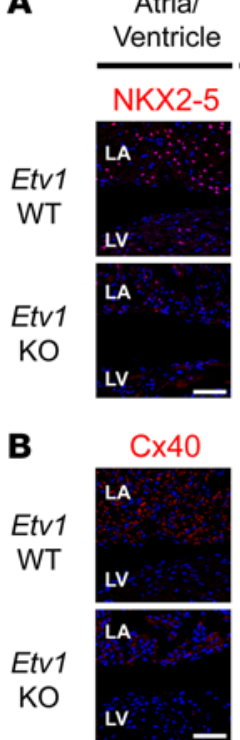

C $\quad \mathrm{Na}_{\mathrm{v}} 1.5$

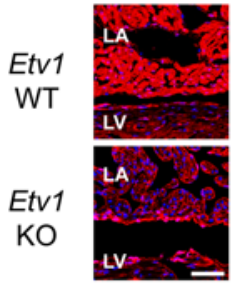

Proximal VCS
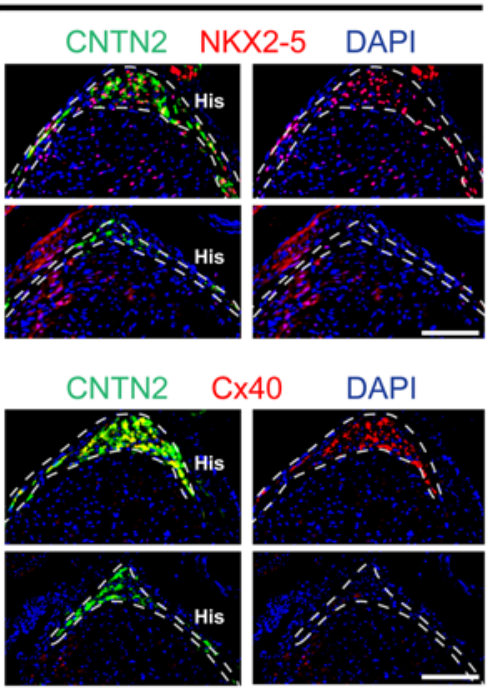

CNTN2 $\quad \mathrm{Na}_{\mathrm{y}} 1.5$
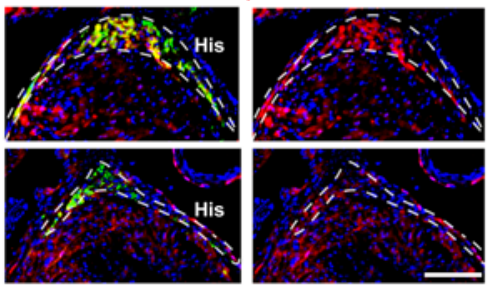

D

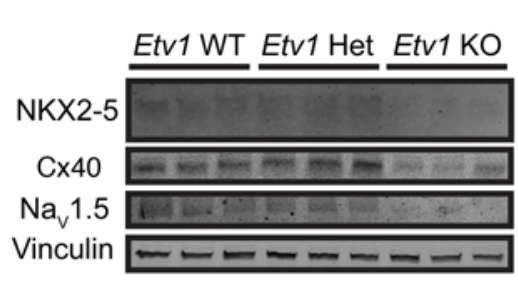

E

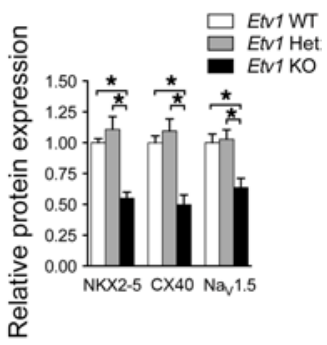

$\mathbf{F}$

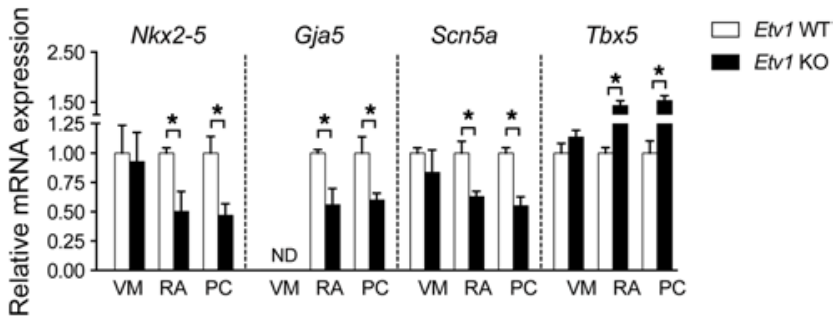

G

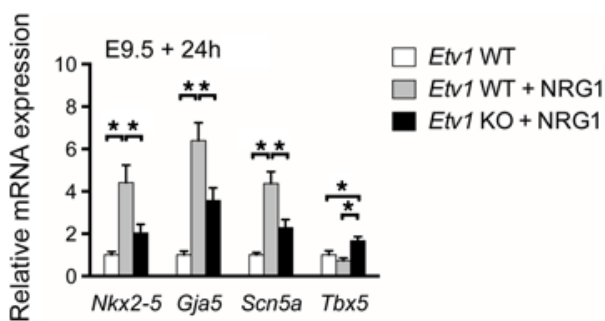

Figure 7. Etv1 KO hearts have reduced expression of fast conduction genes. (A-C) NKX2-5 (A), Cx40 (B), and Na 1.5 (C) expression was evaluated by immunofluorescence in P18 Etv1 WT and KO atria and proximal VCS. VCS cells were identified by CNTN2 expression. Nuclei were stained with DAPI (blue). (D) Western blots of Etv1 WT, Het, and KO atrial tissue lysates detecting NKX2-5, Cx40, and $\mathrm{Na}_{\mathrm{v}} 1.5$. Vinculin was used as loading control. (E) Densitometry quantification of protein levels (normalized to vinculin), displayed relative to WT $(n=6)$. (F) Quantitative RT-PCR of Etv1 WT and KO FACS-purified ventricular, atrial, and Purkinje myocytes detecting fast conduction gene RNA levels (normalized to Gapdh). Relative transcript levels of Nkx2-5, Gja5, Scn5a, and Tbx5, displayed relative to WT $(n=4)$. (C) Quantitative RT-PCR of Nkx2-5, Gja5, Scn5a, and Tbx5 from E9.5 Etv1 WT and KO hearts in culture treated with vehicle control or NRG1 for 24 hours $(n=4)$. ND, not detected. Data represent mean \pm SEM. ${ }^{*} P<0.05,1$-way ANOVA (E and $\left.\mathbf{C}\right)$ or 2-tailed Student's $t$ test $(\mathbf{F})$. Scale bars: $50 \mu \mathrm{m}(\mathbf{A}-\mathbf{C})$.

$\left(\mathrm{V}_{0.5}\right)$ of $\mathrm{I}_{\mathrm{Na}}$ were hyperpolarized in atrial myocytes compared with ventricular myocytes (Figure 9, B and C, and Supplemental Tables 5,7 , and 8). Purkinje cells displayed $V_{0.5}$ activation of $I_{\mathrm{Na}}$ that was similar to that in atrial myocytes, whereas $\mathrm{V}_{0.5}$ inactivation of $\mathrm{I}_{\mathrm{Na}}$ was similar to that in ventricular myocytes (Figure 9, B and C, and Supplemental Tables 5, 7, and 8). Recovery of $\mathrm{I}_{\mathrm{Na}}$ from inactivation was slower to the same degree in atrial and Purkinje myocytes compared with ventricular myocytes (Figure 9D and Supplemental Tables 5 and 9). Moreover, the time-dependent inactivation was faster to the same degree in atrial and Purkinje myocytes compared with ventricular myocytes (Supplemental Figure 11 and Supplemental Tables 5 and 10). Loss of Etv1 resulted in complete homogenization of $\mathrm{I}_{\mathrm{Na}}$ biophysical properties between atrial, Purkinje, and ventricular myocytes (Figure 9, A-D, Supplemental Figure 11, and Supplemental Tables 5-10).

Human PheWAS analysis identifies an association between ETV1 and bundle branch block and heart block. Based on the importance of ETV1 in establishing and maintaining the fast conduction hierarchy in the heart, we next sought to identify associations between ETV1 and human conduction disease. We performed a phenome- wide association (PheWAS) study on the ETV1 SNP rs9639168 (missense, S100G) and 1,804 phenotypes in 26,256 individuals of European ancestry (EA) and 3,269 of African ancestry (AA) from the Vanderbilt BioVU biobank, following previously validated methods (46). The phenotype with the strongest association in AA individuals was left bundle branch block (odds ratio $[\mathrm{OR}]=2.53, P=0.0005$; Figure 10A and Supplemental Table 11). Similarly, "other heart block" (ICD9: 426.6) was the strongest phenotype in EA individuals $(\mathrm{OR}=2.85, P=0.0004$; Figure 10B and Supplemental Table 12). We then evaluated the effect of this SNP on the subset of individuals who also had available ECGs and found similar results. Associations were seen between rs 9639168 and bundle branch block, left bundle branch block, and left anterior fascicular block $(P<0.05$; Supplemental Tables 13 and 14). The difference in the signals between EA and AA populations suggests that rs 9639168 may not be causal but rather be a marker of other variants in ETV1 that likely have different linkage disequilibrium patterns. Interestingly, "lack of coordination" ( $\mathrm{OR}=1.64, P=0.005$, AA), also identified in PheWAS analysis, mirrors the neuromuscular phenotype of Etv1 KO mice, which exhibit severe motor discoordination (38). 
A
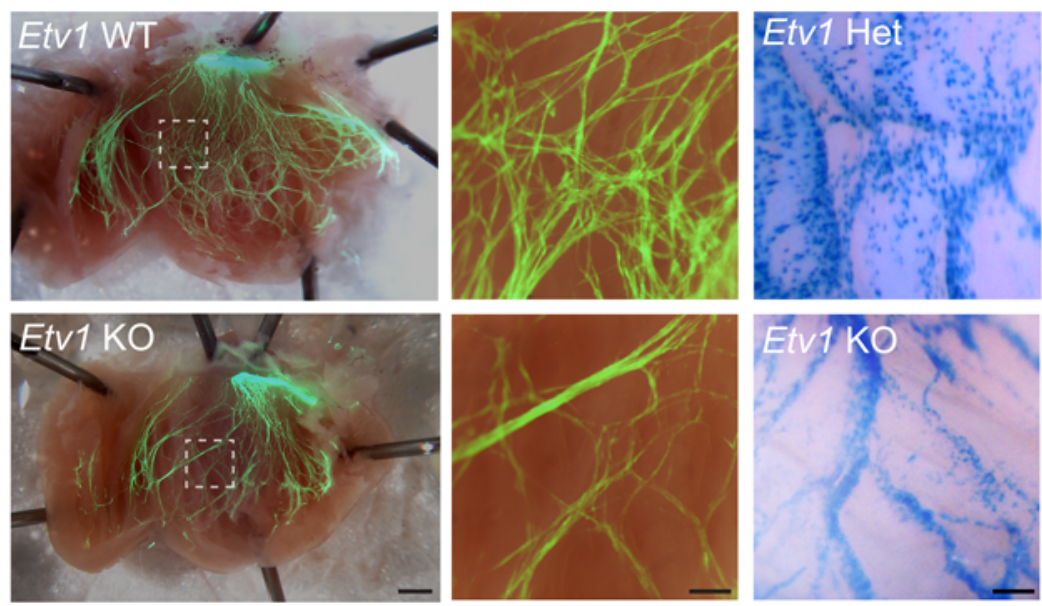

B

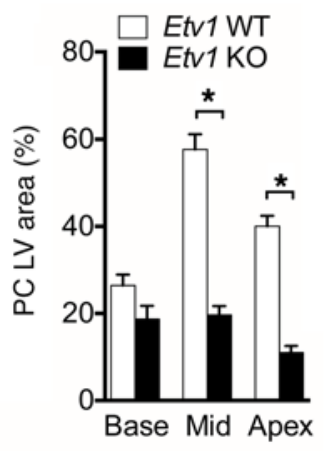

C

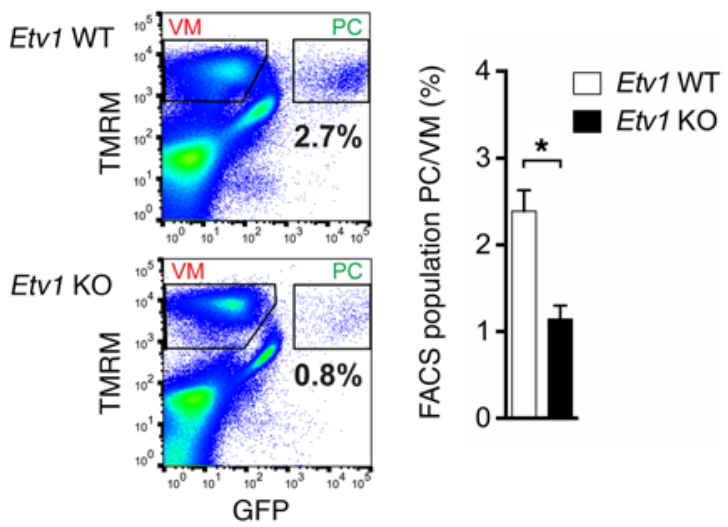

Figure 8. Etv1 KO hearts exhibit hypoplasia of the VCS. (A) P18 Etv1 KO mice backcrossed into Cntn2 ${ }^{E C F P /+}$ reporter line were used to visualize the left VCS. Middle panels are a high-magnification view of the white dashed boxes outlined in the left panels. High-magnification views of X-galstained P18 Etv $7^{n / 2 /+}$ (Het) and Etv $7^{1 / 2 / n / 2}$ (KO) hearts (right panels). (B) Percentage of VCS (CNTN2 ${ }^{\mathrm{ECFP}+}$ ) area was significantly reduced in the left ventricular (LV) mid- and apical regions in Etv1-null hearts $(n=3)$. (C) Dissociated Etv1 WT and KO ventricles stained with the mitochondrial dye tetramethylrhodamine methyl ester perchlorate (TMRM) were purified into ventricular (VM) and Purkinje (PC) fractions. Representative FACS plots of PC (TMRM ${ }^{\text {hi }}$ GFP $\left.^{+}\right)$and VM (TMRM ${ }^{\text {hi }}$ CFP $\left.^{-}\right)$ populations. The PC/VM ratio was quantified for Etv1 WT and KO samples and expressed as a percentage $(n=4)$. Data represent mean \pm SEM. ${ }^{*} P<0.05,2$-tailed Student's $t$ test. Scale bars: 1 $\mathrm{mm}$ (left), $200 \mu \mathrm{m}$ (middle, right) (A).

\section{Discussion}

This study identifies ETV1 as a critical factor essential for transducing endocardially derived NRG1 signals into a transcriptional program responsible for fast conduction gene programming in the heart. ETV1 is a known target of ErbB2 and MAPK signaling pathways and has been implicated in specification, patterning, and functional modulation of various cell types $(37,38,40)$. In the gastrointestinal tract, ETV1 is necessary both for proper development of myenteric and intramuscular interstitial cells of Cajal and for malignant transformation of these cell types into gastrointestinal stromal tumors (47). In the central nervous system, ETV1 has been shown to regulate terminal differentiation of cerebellar granule cells (39) and dopaminergic neurons (48). ETV1 has a direct role in establishing the sensory-motor circuitry in the developing spinal cord (38) and also dynamically modulates the electrophysiological properties of postmitotic fast-spiking interneurons through transcriptional regulation of $\mathrm{K}_{\mathrm{v}} 1.1$ channels (37), the activity of which is also known to be regulated by NRG1/ErbB4 (49). Our data now implicate ETV1 as a major regulator of cardiac conduction biology and place it in the context of NRG1 signaling in the heart. Through NRG1-dependent activation of the Ras-MAPK pathway, ETV1 expression and activity are confined to subendocardial atrial and Purkinje myocytes, where ETV1 orchestrates the expression of the fast conduction gene program.

Etv1 expression is tightly regulated during development in a tissue-specific manner. High levels of ETV1 are detectable in the heart, brain, and lung, while expression levels are low in skeletal muscle and liver $(50,51)$. In mammalian and amphibian tissues Etv1 expression is regulated by peptide growth factors $(52,53)$. In Xenopus animal caps, the peptide growth factors fibroblast growth factor (FGF), bone morphogenetic protein 4 (BMP4), and activin were all able to drive transcription of etv1 (53). During murine cerebral cortex development, Etv1 expression is regulated by FGF8 signaling in Cajal-Retzius cells in the rostral telencephalon (52). In human and murine breast cancer cells, ETV1 expression levels correlate with ErbB2 overexpression and malignant transformation $(32,54)$. Therefore, the identification of NRG1 as an induction agent for Etv1 in mammalian hearts is in keeping with its known regulation in other tissue types and ensures that Etv1 expression is confined to cardiac regions with high levels of NRG1 exposure, namely the PAM and VCS. Interestingly, ETV1 has been shown in some cellular contexts to induce expression of ErbB2 (55), providing a potential mechanism for reinforcing NRG1-dependent signaling in these myocardial regions.

Activation of NRG1 and MAPK signaling has been shown to positively correlate with fast conduction gene expression in primary and stem cell-derived cardiomyocytes as well as in embryonic heart culture assays (56-59). Conversely, blockade of NRG1 signaling was associated with reduced levels of $\operatorname{Scn} 5 a$ transcript in human embryonic stem cell-derived cardiomyocytes (59). Our work validates these findings and places them in the context of an NRG1-MAPK-RSK/MSK signaling pathway that converges on ETV1 to drive enrichment of Nkx2-5, Gja5, and $S c n 5 a$ in a tissue-specific manner. NRG1 signaling achieves 
A

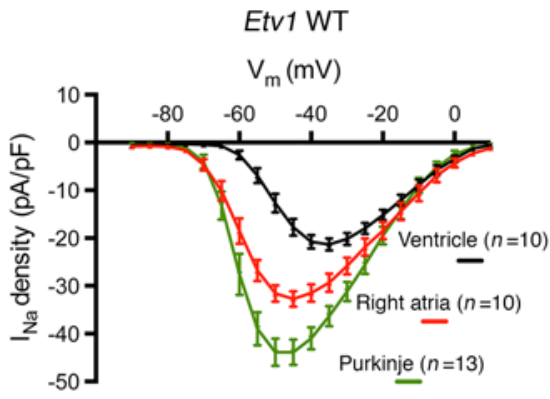

B

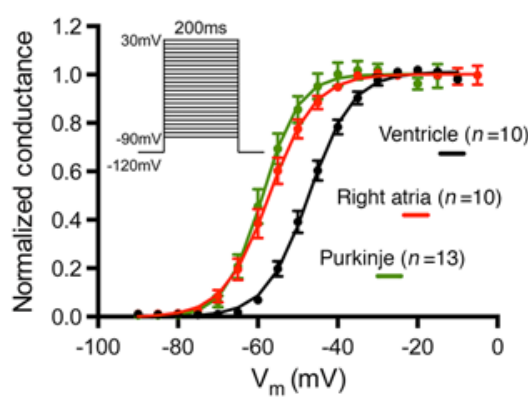

C

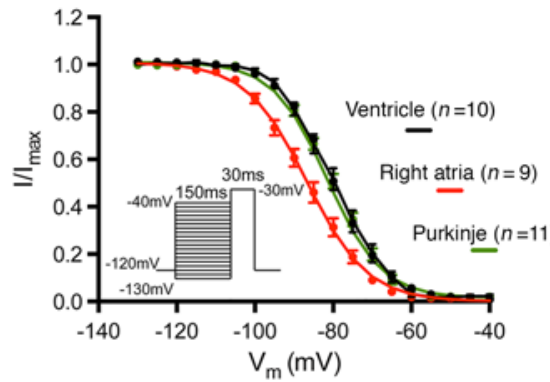

D

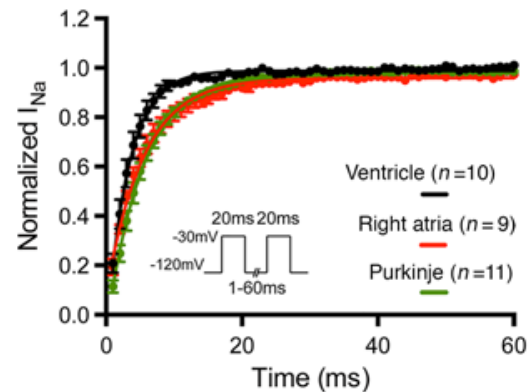

\section{Etv1 KO}
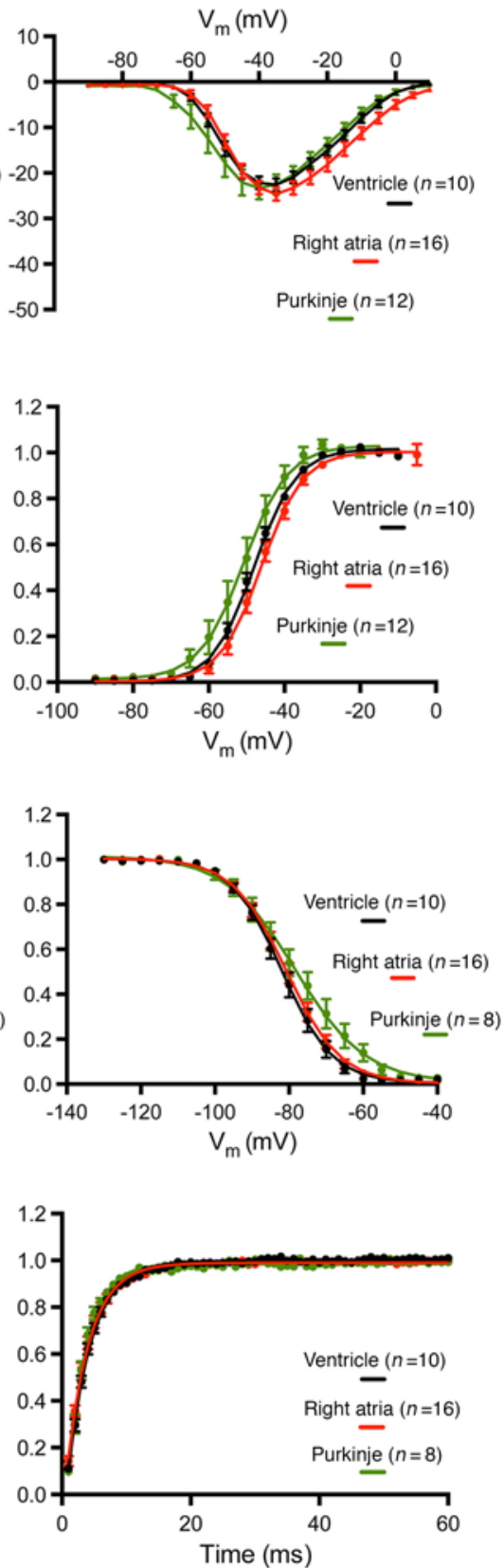
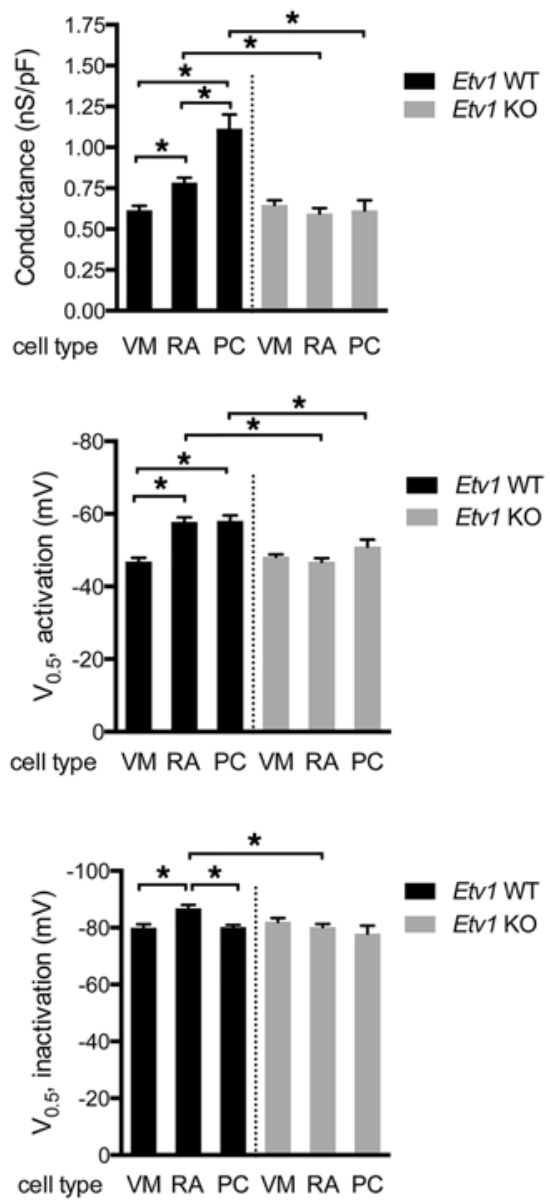

cell type VM RA PC VM RA PC

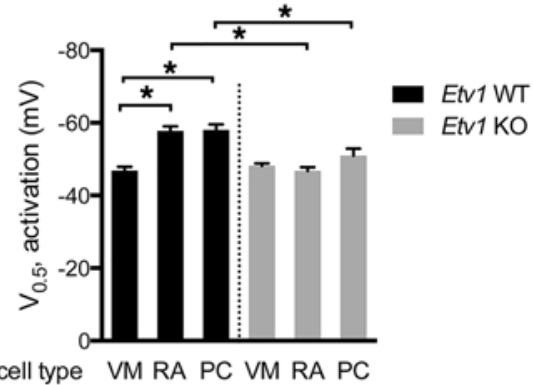

cell type VM RA PC VM RA PC

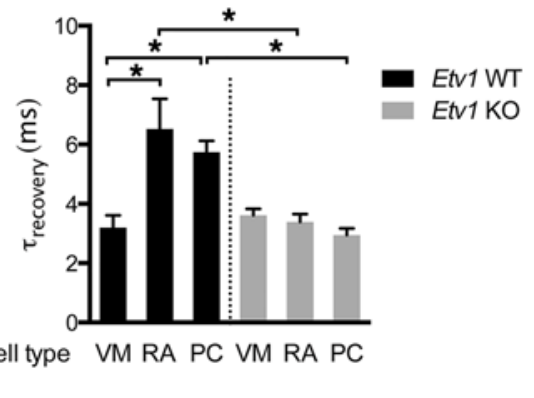

Figure 9. Loss of Etv1 homogenizes sodium channel biophysical properties between ventricular, atrial, and Purkinje myocytes. Whole-cell patch clamp was performed on dissociated cardiac cells (ventricular, atrial, Purkinje myocytes) from P18 Etv1 WT and KO mice in a Cntn2 $2^{E C F P /+}$ background. (A) Comparison of sodium current-voltage (I-V) relationship. (B) Voltage dependence of steady-state activation. (C) Voltage dependence of steady-state inactivation. (D) Time course of recovery from inactivation. (A-D) Maximum conductance, voltage at half activation $\left(\mathrm{V}_{0.5}\right.$, activation), voltage at half inactivation $\left(\mathrm{V}_{0.5}\right.$, inactivation), and tau of recovery ( $\tau_{\text {recovery }}$ ) were used to assess significant differences among experimental groups (right panels), respectively $(n=4$ hearts) Diagrams outlining patch clamp protocols are included for each endpoint. Data represent mean \pm SEM. ${ }^{*} P<0.05,1$-way ANOVA.

highly restricted enrichment of these fast conduction genes in PAM and VCS myocytes through dual regulation of ETV1 expression level and phosphorylation state.

When placed in the context of previously published work from Nkx2-5 mutant mouse models, our data indicate that ETV1 orchestrates fast conduction physiology in atrial and Purkinje myocytes through Nkx2-5-dependent and -independent mechanisms. Etv1 KO hearts displayed an approximately 50\% reduc- tion in Nkx2-5 RNA and protein levels specifically in atrial and Purkinje myocytes, which resulted in a corresponding approximately 50\% reduction in Cx40 levels and VCS hypoplasia. $\mathrm{Cx} 40$ is a known direct transcriptional target of NKX2-5 $(8,60)$ and responds in a dose-dependent manner (9). Etv1-null mice displayed structural and functional defects of the VCS that phenocopy Nkx2-5 haploinsufficient mice $(18,44)$. Meysen et al. attributed the functional defects of the VCS in $N k \times 2-5^{+/}$mice to 

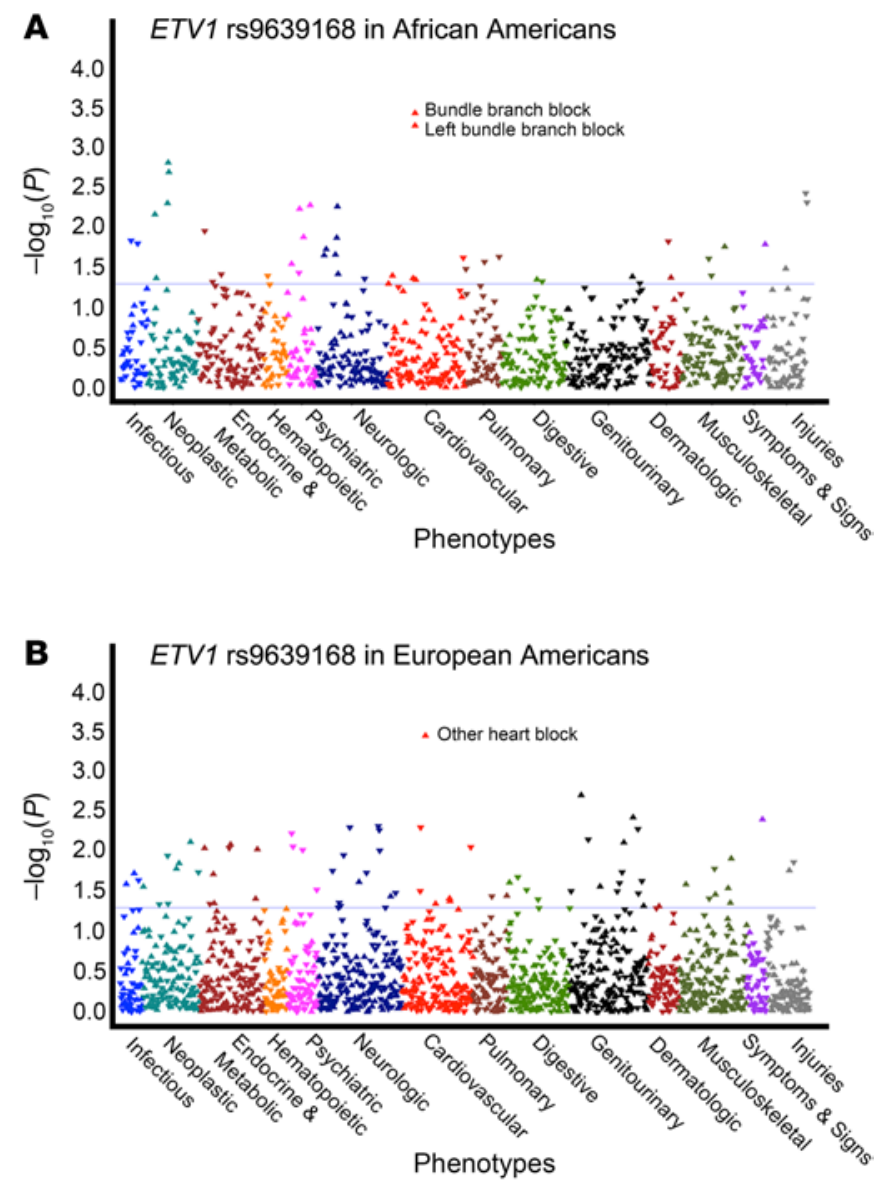

the morphological abnormalities of the His-Purkinje system, as mutant Purkinje cells displayed normal action potential properties (44). In agreement with these findings, a perinatal Nkx2-5 KO mouse model displayed normal levels of $S c n 5 a$ in atrial and Purkinje myocytes (19). In contrast, Etv1 KO mice displayed a reduction of $S c n 5 a$ RNA and protein levels in atrial and Purkinje myocytes, resulting in homogenization of $\mathrm{Na}_{\mathrm{v}} 1.5$ throughout the heart. The loss of cell type-specific biophysical properties of the sodium current in Etv1 KO hearts suggests that ETV1 regulates $S c n 5 a$ and other genes known to modulate the sodium current. Thus, the observed conduction slowing in Etv1 KO hearts is likely to reflect alterations in active (ionic) and passive (gap junctional) conductance as well as VCS hypoplasia in the His-Purkinje system.

The identification of associations between an ETV1 sequence variant (rs9639168) and bundle branch block in African Americans and heart block in European Americans points to a potential role of ETV1 in fast conduction gene programming in humans. Whether rs9639168 directly or indirectly affects ETV1 function or instead modifies ETV1 expression levels remains to be determined. The observation that NRG1 signaling blockade downregulates Etv1 expression and results in conduction abnormalities in postnatal hearts points to a continued dependency of NRG1 signaling to maintain the ETV1 fast conduction gene program. Whether there is an age-dependent degradation of the NRG1ETV1 signaling axis that ultimately plays a role in senile conduction disease will be particularly interesting.
Figure 10. PheWAS plot for ETV1 rs9639168 shows significant association between ETV1 and cardiac conduction disease in African Americans and European Americans. Each panel represents 1,804 phenotypes tested for association with ETV1 rs9639168, using logistic regression assuming an additive genetic model adjusted for age, sex, study site, and the first 3 principal components. Phenotypes were grouped along the $x$ axis by categorization within the PheWAS code hierarchy. (A) PheWAS associations for rs 9639168 for ETV1 in African Americans $(n=3,269)$. The most significant association was bundle branch block (OR $=2.13, P=0.00037)$. (B) PheWAS associations for rs9639168 for ETV1 in European Americans $(n=26,256)$. The most significant association was other heart block ( $\mathrm{OR}=2.85, P=0.00036)$.

In summary, using multiple orthogonal approaches, including signal transduction screens, transcriptional profiling, mouse genetic models, cellular electrophysiology, and human genetic analysis, we discovered an NRG1 signaling axis that drives cell type-specific expression and activation of ETV1, conferring a fast conduction phenotype in atrial and Purkinje myocytes. Our studies identify ETV1 as a critical regulator of the fast conduction phenotype and demonstrate the biological importance of this gene in cardiac conduction disease.

\section{Methods}

Mutant mice. CCS-lacZ (21), Cntn2-EGFP BAC transgenic (30), and Etv1-nlz (38) (provided by Thomas Jessell, Columbia University, New York, New York, USA) mutant mice have all been previously described. CCS-lacZ and Cntn2-EGFP mice were maintained in a CD1 genetic background. Etv1-null mice were maintained in a C57BL/6 background. For Purkinje cell morphology imaging and quantification, the Etv1 ${ }^{\text {nlz }}$ mouse line was bred into the Cntn2-EGFP background. For functional studies, Etv1 ${ }^{\text {nlz }}$ Cntn $2^{\text {EGFP }}$ mouse lines were backcrossed more than 5 generations into the C57BL/6 background.

Antibody reagents. Immunofluorescent antibodies were [target, dilution (species, company, product number)]: NKX2-5, 1:100 (rabbit, Abcam, ab91196); ETV1, 1:100 (rabbit, Abcam, ab36788); ETV1, 1:200 (goat, Santa Cruz Biotechnology, sc-1953); IRX3, 1:100 (rabbit, Abcam, ab25703); $\mathrm{Na}_{\mathrm{v}} 1.5$, 1:50 (rabbit, Alomone Labs, ASC005); Cx40, 1:250 (rabbit, Alpha Diagnostic, Cx40A); CNTN2, 1:40 (goat, R\&D Systems, AF4439); $\alpha$-actinin (sarcomeric), 1:100 (mouse, Sigma-Aldrich, A7811); phospho-ErbB2, 1:100 (rabbit, Abcam, ab108371); phospho-ErbB4, 1:100 (rabbit, Abcam, ab109273); phospho-p44/42 MAPK (pErk1/2), 1:100 (rabbit, Cell Signaling, 4370S); and mouse anti-phospho-histone H3 (PHH3), 1:100 (mouse, Abcam, 14955). Secondary antibodies were donkey anti-rabbit, 1:500 (Santa Cruz Biotechnology, sc-2784); donkey anti-goat, 1:500 (Santa Cruz Biotechnology, sc-2024); and donkey anti-mouse, 1:500 (Santa Cruz Biotechnology, sc-2099).

Western blot primary antibodies were [target, dilution (species, company, product number)] Nkx2-5, 1:1,000 (mouse, Abcam, ab91196); ETV1, 1:200 (goat, Santa Cruz Biotechnology, sc-1953); antiphosphoserine, 1:500 (rabbit, Millipore, AB1603); $\mathrm{Na}_{\mathrm{v}}$ 1.5, 1:500 (rabbit, Alomone Labs, ASC-005); Cx40, 1:1,000 (rabbit, Alpha Diagnostic, Cx40A); and vinculin, 1:5,000 (mouse, Abcam, ab11194). Secondary antibodies were goat anti-rabbit, 1:15,000 (LI-COR, 926-32211); goat anti-mouse, 1:15,000 (LI-COR, 926-32220); and donkey anti-goat, 1:15,000 (LI-COR, 926-32214).

Embryonic heart culture assays and inhibitor assay. E9.5 CCS-lacZ hearts were harvested and cultured in DMEM containing 1\% FBS, 
penicillin, and streptomycin (GIBCO/Invitrogen) in 24-well culture plates. The recombinant peptide containing the $\beta$ variant of the epidermal growth factor-like domain of NRG1 (R\&D Systems) was added to each well at a final concentration of $2.5 \times 10^{-9} \mathrm{M}$ (20). Lyophilized NRG1 was reconstituted at $100 \mu \mathrm{g} / \mathrm{ml}$ in sterile PBS. The control group received vehicle (sterile PBS) only. Medium in both conditions was replaced every 12 hours. Cultures were maintained for up to 48 hours. Nonbeating cultures were excluded from analysis.

For kinase inhibitor studies, the following conditions were used: (a) vehicle control, (b) NRG1 alone, (c) kinase inhibitor alone, or (d) NRG1 plus kinase inhibitor. Doses used for kinase inhibitor studies were: PP2 $(10 \mu \mathrm{M})(61)$, LY294002 $(25 \mu \mathrm{M})(62,63)$, PD98059 $(50 \mu \mathrm{M})(64)$, FR180204 $(20 \mu \mathrm{M})(65,66)$, SL0101 $(50 \mu \mathrm{M})(67), \mathrm{H} 89(10 \mu \mathrm{M})(68)$.

For RNA analysis, E9.5 heart culture assays described above were used to perform qPCR. Four embryonic hearts were pooled within each replicate to increase total RNA recovered and to minimize assay variability.

Whole-mount staining for $\beta$-galactosidase activity. Tissues were collected in ice-cold PBS and fixed for 15 minutes (embryonic) or 1 hour (postnatal) in fix solution (2\% formaldehyde, $0.2 \%$ glutaraldehyde, $0.02 \%$ NP-40, 0.01\% sodium deoxycholate in PBS). After fixation, tissues were rinsed in PBS 3 times and then stained overnight at $37^{\circ} \mathrm{C}$ in the dark with stain solution $\left[5 \mathrm{mM} \mathrm{K}_{3} \mathrm{Fe}(\mathrm{CN})_{6}, 5 \mathrm{mM} \mathrm{K}_{4} \mathrm{Fe}(\mathrm{CN})_{6}, 1\right.$ $\mathrm{mg} / \mathrm{ml}$ 5-bromo-4-chloro-3-indolyl- $\beta$-D-galactopyranoside (X-gal), $2 \mathrm{mM} \mathrm{MgCl}, 0.02 \% \mathrm{NP}-40,0.01 \%$ sodium deoxycholate in PBS]. Bright-field images of hearts were taken using the Zeiss Discovery V8 microscope equipped with a Zeiss AxioCam Color camera interfaced with Zeiss Zen 2012 software.

Immunohistochemistry. Adult hearts were excised and immediately perfusion-fixed in $4 \%$ paraformaldehyde overnight. Embryonic hearts were excised and immediately fixed in $4 \%$ paraformaldehyde for 2 hours. Samples were then washed in ice-cold PBS and equilibrated in $30 \%$ sucrose at $4^{\circ} \mathrm{C}$ overnight. The samples were then embedded into Tissue-Tek OCT compound (Fisher Scientific), and frozen tissues (10- $\mu \mathrm{m}$ sections) were cut and collected on Superfrost Plus microscope slides (Fisher Scientific). Sections were blocked with $10 \%$ serum and $0.01 \%$ Triton in PBS for 1 hour, then incubated with primary antibodies overnight. Sections were then washed in PBS and incubated with secondary antibodies with Alexa Fluor dyes (Invitrogen) for 1 hour before mounting. Slides were coverslipped with Vectashield mounting medium with DAPI (Vector Laboratories). Stained sections were visualized on an Axiovert 200M fluorescence microscope. Images were collected using uniform exposure settings for each staining run on an AxioCam camera with AxioVision 4.48 software (Carl Zeiss). Confocal images were taken with a Leica TCS SP5 confocal microscope using Leica LAS AF acquisition software. Quantification of pHH3-positive nuclei was performed per high-power field $(\times 40)$ in trabecular and compact myocardial regions using Image J software (NIH).

Laser capture microdissection and RNA extraction. Laser capture microdissection (LCM) was performed as previously described except for the following modifications (69). E10.5 and E12.5 embryonic hearts were collected in ice-cold PBS, immediately placed into OCT compound, and frozen at $-80^{\circ} \mathrm{C}$. Cryosections ( $9 \mu \mathrm{m}$ thickness) were subjected to $\mathrm{H} \& \mathrm{E}$ staining and subsequently dehydrated in graded ethanol solutions (95\% twice, 5 minutes, 100\% 3 times, 5 minutes) and cleared in xylene ( 3 times, 5 minutes). After air-drying of the sam- ples for 30 minutes, LCM was performed under direct microscopic visualization. Trabecular versus compact myocardial cells were collected onto thermoplastic film on LCM caps (Arcturus Engineering). RNA was extracted from cells captured onto thermoplastic film using the PicoPure RNA Isolation Kit (Arcturus Engineering). Differential expression libraries were generated using Affymetrix gene chips, as previously described (29).

Cardiomyocyte enzymatic dissociation for whole-cell patch clamp and FACS purification. Cardiac cells were dissociated from neonatal or adult $C n t n 2^{E G F P /+}$ hearts. Hearts were Langendorff-perfused and enzymatically digested as previously described (70). Myocyte FACS-based purification was performed as previously described (43). Briefly, the mitochondrial dye tetramethylrhodamine methyl ester perchlorate (TMRM; Invitrogen) was used to identify cardiomyocytes. Fifty-nanomolar TMRM was incubated with dissociated single-cell ventricular cardiomyocytes for 15 minutes using Cntn2 $2^{\text {EGFP/+ }}$ hearts. Cell suspensions were purified by FACS (Beckman Coulter MoFlo). Purkinje cell $\left(\mathrm{TMRM}^{\mathrm{hi}} \mathrm{GFP}^{+}\right)$and ventricular myocyte $\left(\mathrm{TMRM}^{\mathrm{hi}} \mathrm{GFP}^{-}\right)$populations were collected. RNA was isolated from sorted cell populations using the PicoPure RNA Isolation Kit (Arcturus Engineering).

Whole-cell $I_{\mathrm{Na}}$ recordings. All $\mathrm{I}_{\mathrm{Na}}$ recordings in isolated cardiomyocytes were conducted in whole-cell configuration at room temperature. Recording pipettes were filled with a solution containing (in $\mathrm{mM}$ ) $\mathrm{NaCl}$ 5, CsF 135, EGTA 10, MgATP 5, HEPES 15, pH 7.2, with CsOH. Cells were maintained in a solution containing (in $\mathrm{mM}$ ) $\mathrm{NaCl} 5, \mathrm{CsCl}$ 112.5, TEACl 20, $\mathrm{CdCl}_{2}$ 0.1, $\mathrm{MgCl}_{2} 1, \mathrm{CaCl}_{2} 1$, HEPES 20, glucose 11, $\mathrm{pH}$ 7.4, with $\mathrm{CsOH}$. To determine the peak current voltage relation, 200-ms voltage pulses were applied to membrane potential $\left(\mathrm{V}_{\mathrm{m}}\right)-90$ $\mathrm{mV}$ to $+30 \mathrm{mV}$ in 5-mV voltage steps, from a holding potential of $\mathrm{V}_{\mathrm{m}}=$ $-120 \mathrm{mV}$. The interval between voltage steps was 3 seconds. For analysis of steady-state activation, $\mathrm{I}_{\mathrm{Na}}$ was normalized to the driving force and maximal conductance. The normalized conductance was then plotted against $\mathrm{V}_{\mathrm{m}}$. The steady-state voltage-dependent activation curves were fitted to the Boltzmann's function. Steady-state inactivation was determined by stepping $\mathrm{V}_{\mathrm{m}}$ from $-130 \mathrm{mV}$ to $-40 \mathrm{mV}$, followed by a $30-\mathrm{ms}$ test pulse to $\mathrm{V}_{\mathrm{m}}=-30 \mathrm{mV}$ to elicit $\mathrm{I}_{\mathrm{Na}}$. The steady-state voltage-dependent inactivation curves were fitted to Boltzmann's functions. Recovery from inactivation was studied by the application of paired voltage clamp steps. Two 20-ms test pulses (S1, S2) to $\mathrm{V}_{\mathrm{m}}=$ $-30 \mathrm{mV}$ (holding potential $=-120 \mathrm{mV}$ ) were separated by increasing increments of $1 \mathrm{~ms}$ to a maximum S1-S2 interval of $60 \mathrm{~ms}$. The S1-S1 interval was kept constant at 3 seconds. The time-dependent recovery-from-inactivation curves were fit with exponential functions. All recordings were obtained using an Axon multiclamp 700B Amplifier coupled to a pClamp system (version 10.2, Axon Instruments).

Transcriptional profiling and quantitative RT-PCR. Isolated RNA from LCM samples or FACS-purified cells was amplified using the Ovation Pico WTA System V2 (NuGEN). Differential gene expression analysis was performed using Mouse Genome 430 2.0 Array (Affymetrix). All original microarray data were deposited in the NCBI's Gene Expression Omnibus (GEO GSE60987). Data analysis was performed using robust multiarray average (RMA) followed by linear models for microarray data (LIMMA) to identify differentially expressed transcripts with an FDR of 0.05. Quantitative RT-PCR (qPCR) was performed on cDNA using the Quantitect SYBR Green PCR Kit (Qiagen). All qPCR probes were purchased though Origene and performed according to the manufacturer's protocol. 
Western blot analysis. For atrial samples, left and right atrial samples were collected from P18 mice and immediately cryopreserved in liquid nitrogen. Atria were then homogenized in RIPA buffer containing protease and phosphatase inhibitors ( $150 \mathrm{mM} \mathrm{NaCl}, 1.0 \% \mathrm{NP}-40$ or $0.1 \%$ Triton X-100, 0.5\% sodium deoxycholate, $0.1 \%$ sodium dodecyl sulphate, $50 \mathrm{mM}$ Tris- $\mathrm{HCl}, \mathrm{pH} 8.0$, and protease and phosphatase inhibitors). Samples were run on $10 \%$ precast polyacrylamide gradient gels (Invitrogen) and transferred to nitrocellulose (Bio-Rad) overnight at $4^{\circ} \mathrm{C}$. Nitrocellulose membranes were incubated in blocking buffer consisting of PBS with Tween-20 (0.05\%) and 5\% nonfat dry milk. Membranes were then incubated with specific primary antibodies diluted in $5 \%$ nonfat dry milk overnight at $4^{\circ} \mathrm{C}$ followed by wash steps and secondary antibodies (LI-COR). Antigen complexes were visualized and quantified with the Odyssey Imaging System (LI-COR).

Cultured rat atrial myocytes. Enriched P1 rat atrial myocyte cultures were obtained by enzymatic digestion (trypsin $2.5 \%$ /collagenase $1 \%$ ) as previously described (71). After overnight plating in DMEM plus 10\% FBS plus supplements (3 mM pyruvic acid, $2 \mathrm{~g} / 1 \mathrm{BSA}$, 0.5 $\mathrm{mg} / \mathrm{ml}$ primocin, $15 \mathrm{mM}$ HEPES, $4 \mu \mathrm{g} / \mathrm{ml}$ transferrin, $0.7 \mathrm{ng} / \mathrm{ml}$ sodium selenite, $5 \mu \mathrm{g} / \mathrm{ml}$ linoleic acid, $10 \mu \mathrm{M}$ ascorbic acid), cells were maintained in serum-free DMEM with supplements. The final myocyte cultures contained more than $90 \%$ atrial myocytes at a density of $2 \times 10^{6}$ cells per 60 -mm dish. NRG1 experiments were performed 24 hours after plating. Cells were used for either ETV1 immunoprecipitation assays or immunofluorescent staining after 0,1 , or 3 hours of vehicle or NRG1 treatment. Quantification of ETV1 nuclear accumulation in dissociated neonatal rat myocytes was performed per cell using Image J software. DAPI channel was used to define total nuclear area.

ETV1 immunoprecipitation assay. Atrial myocyte cells were washed twice with ice-cold PBS and resuspended in $1 \mathrm{ml}$ lysis buffer $(10 \mathrm{mM}$ Tris, pH 8, 1 mM EDTA, 0.5 mM EGTA, 0.5\% $N$-lauroyl sarcosine, and protease and phosphatase inhibitors) per 60-mm dishes. Each 1-ml cell resuspension was individually homogenized on ice using Dounce homogenizers (types A and B). Dynabeads M-280 sheep anti-rabbit IgG (Life Technologies, 11203D) was used with ETV1 1:100 (rabbit, Abcam, ab36788) according to the manufacture-recommended protocol. Western blot analysis was performed using anti-phosphoserine (rabbit) and anti-ETV1 (goat) antibodies. Protein levels were quantified by densitometry (normalized to ETV1) and plotted relative to WT at baseline.

Electrocardiograms. Surface ECGs were obtained using subcutaneous electrodes attached at the 4 limbs, as previously described (72). P18 mice were anesthetized with inhaled $2 \%$ isoflurane. Heart rate was monitored, and core body temperature was maintained at $37.5^{\circ} \mathrm{C}$ using a heat lamp. ECG analysis was performed in an unbiased fashion where 200 beats were analyzed using LabChart 7 Pro version 7.3.1 (ADInstruments Inc.). Detection and analysis of P wave, PR interval, QRS wave, and QT intervals were set to mouse ECG parameters. Mice with heart rates below $400 \mathrm{bpm}$ were excluded from the analysis.

Intracardiac electrograms were obtained with a Millar EPR-800 1.1Fr octapolar catheter (ADInstruments Inc.) for bipolar recordings at the level of the His bundle. P18 mice were anesthetized with inhaled $1 \%-1.5 \%$ isoflurane. Heart rate was monitored, and core body temperature was maintained at $37.5^{\circ} \mathrm{C}$ using a heat lamp. Access was via the right internal jugular vein. The 8 electrodes spaced $1 \mathrm{~mm}$ apart directly contact the endocardial surface of the heart. Intracardiac measurements were performed as follows: (a) His bundle electrograms were measured when the atrial, His, and ventricular electro- grams were stable over more than 25 beats. (b) Measurement of the $\mathrm{AH}$ interval was taken from the His bundle recording from the onset of deflection from baseline of the local atrial electrogram to the onset of deflection from baseline of the His bundle electrogram. (c) Measurement of the HV interval was taken from the onset of deflection from baseline of the His bundle electrogram to the onset of deflection from baseline of the earliest ventricular electrogram, whether on surface ECG or intracardiac recordings.

Transthoracic echocardiography. Echocardiography was performed using the Vevo 2100 high-resolution ultrasound imaging system with a real-time 30-MHz linear array scanhead (MS400) at a frame rate of 235 fps, a focal length of $8 \mathrm{~mm}$, and a 10-by-10-mm field of view (VisualSonics). Mice were anesthetized with $2 \%$ isoflurane, and hair was removed from the chest using a depilatory cream (Nair, Church \& Dwight Co. Inc.). Warmed ultrasound transmission gel was placed on the chest and used to obtain left ventricular endpoints of cardiac function. B-mode cardiac imaging was conducted on transverse (short-axis) plane. The papillary muscles were used for the short axis imaging landmark. M-mode recordings of the left ventricle were also recorded at the short-axis B-mode imaging plane to obtain left ventricular function and dimensions through the cardiac cycle. Heart rate was monitored, and core body temperature was maintained at $37.5^{\circ} \mathrm{C}$ using a heated platform and hair dryer throughout the procedure. Mice with heart rates below 400 bpm were excluded from the analysis. Data analysis was performed on VisualSonics Vevo 2100 V1.5.0 software. The following parameters were measured using short axis M-mode: diastolic and systolic left ventricular internal diameter, anterior wall thickness, and posterior wall thickness. From these measurements, left ventricular ejection fraction and percent fractional shortening were calculated within the Vevo software.

VCS whole-mount quantification. Purkinje cell imaging and quantification of EGFP fluorescence were conducted using Cntn $2^{E G F P /+}$ reporter mice. Etv1 ${ }^{n l z /+} \mathrm{Cntn} 2^{\mathrm{EGFP} /+}$ mice were generated to study the spatial and temporal expression of Etv1 within the cardiac conduction system as reported by EGFP. Hearts were excised, immediately placed in ice-cold PBS, and fixed for 1 hour in the $\beta$-galactosidase fixative solution (see above). For imaging of the left VCS, the left ventricular wall was cut open at the center of the free wall. Free wall edges were pinned down using 30-gauge needles to expose the left ventricular septum. For imaging of the right VCS, the anterior portion of the right ventricular free wall adjacent to the septum was cut to expose the right ventricular septum and free wall Purkinje fiber network. Bright-field and fluorescent images of the hearts were taken using the Zeiss M2Bio microscope equipped with a Zeiss AxioCam Color camera interfaced with Zeiss AxioVision 2012 software. To acquire EGFP- and X-galstained composite images, EGFP images were first acquired, and then pinned hearts underwent $\mathrm{X}$-gal staining and were imaged again. Overlay of bright-field and fluorescent channels was done using Photoshop CS6. Littermates were imaged on the same day at comparable magnification, exposure, and light intensity. Area of $\mathrm{GFP}^{+}$Purkinje cells was measured using ImageJ software in regions on the left ventricular septum, right ventricular septum, and right ventricular free wall. Quantification was normalized to total area as specified.

ErbB2 inhibitor studies. P1 Etv1 ${ }^{n z /+}$ mice were treated with either vehicle (33\% DMSO in sterile saline) or AG825 (1 mg/ kg) i.p. daily for 7 days. P1 pups were randomly assigned to either the treatment or vehicle group. ECGs were obtained and hearts were X-gal-stained using methods described above. 
Phenome-wide association study. To investigate possible human associations with ETV1 variation, we used a population of 26,256 adults (age $>18$ years) of European ancestry (EA) and 3,269 of African ancestry (AA) who had genotyping on Illumina HumanExome BeadChip version 1.1 and available electronic health record (EHR) data from the Vanderbilt BioVU DNA biobank (73). This platform contained 1 SNP, rs9639168, in ETV1, which corresponds to a serine to glycine in multiple splice variants. The minor C allele of rs9639168 was present in $34 \%$ of EA and $19 \%$ of AA individuals. We then evaluated all phenotypes defined using a phenome-wide association study (PheWAS) of this SNP in EA and AA individuals using previously described methods (46). Briefly, the method defines cases for more than 1,600 phenotypes by the presence of specific International Classification of Diseases, 9th edition (ICD9), codes on at least 2 different days. Controls for each phenotype are defined as individuals who lack case ICD9 codes and other codes that are related. For example, cases of the "bundle branch block" phenotype are defined with 426.5, 426.50, 426.53, and 426.54, while its controls are defined as absence of the 426-427.99 range of ICD9 codes. We used version 1.2 of the PheWAS code terminology system and the R PheWAS (74) package to calculate the PheWAS and graph results, both of which can be downloaded from http://phewascatalog. org. We used logistic regression for each phenotype with 20 cases or more, adjusted for age and sex, assuming an additive genetic model.

Analyzing human ECG phenotypes. After seeing that the PheWAS identified cardiac conduction phenotypes, we then followed up by analyzing these phenotypes as documented on ECGs available in the EHR. We extracted keywords for left and right bundle branch block, left anterior and posterior fascicular blocks, and all bundle branch blocks from the narrative text ECG impressions, which are generated via interaction of the ECG system and cardiologists. Fifty reports for each type of block were reviewed without identifying any false positives. Cases of each phenotype are defined by the corresponding keywords' having ever been present in their ECG report. We identify 1 control group for all phenotypes, defined as individuals with at least 1 ECG report in their EHR and absence of any aforementioned keywords of any phenotypes. We used logistic regression to study the association of the C allele of rs9639168 with each ECG phenotype, adjusted for age at the last ECG, sex, and number of ECG reports in the EHR. Specific regular expressions used to define each bundle branch block are defined in Supplemental Table 13.

Statistics. Endpoints were compared using 1-way ANOVA or 2-tailed Student's $t$ test where appropriate. $P$ less than 0.05 was considered statistically significant. Sample size calculations were done using preliminary data to design the experiment for measuring continuous variables. Groups were constructed to detect a 30\% difference between experimental and control groups with a power of $90 \%$ and a significance level of 0.05. Mean and SEM were reported for each group. Experimental groups were blinded until the endpoints were analyzed. Animal studies were done prior to genotyping, ensuring blinded observations. All animal ECGs and ultrasound data analysis were conducted by 2 operators.

Study approval. All protocols conformed to the Association for the Assessment and Accreditation of Laboratory Animal Care and the NYU School of Medicine Animal Care and Use Committee. Protocols for studies of cadaveric fetal human tissues were approved by the NYU Institutional Review Board.

\section{Author contributions}

AS, GIF, and DSP conceived the project, designed the experiments, analyzed the data, and wrote the manuscript. XL carried out cell electrophysiological experiments, under the advice and supervision of MD. HM, LB, JCD, NJC, and DMR carried out PheWAS experiments. AS, FYL, JZ, and DSP performed all other experimental work.

\section{Acknowledgments}

This work was supported by grants from the NIH to AS (T32 GM066704, F31 HL132438), MD (R01 GM57691), and GIF (R01 HL105983). Additional support for Vanderbilt's participation in the Electronic Medical Records and Genomics (eMERGE) network comes from the National Institute for General Medical Sciences (P50-GM115305), National Library of Medicine (R01-LM01068), and National Human Genome Research Institute (HG004603, HG006378, HG008672). BioVU is supported by the National Center for Research Resources (UL1 RR024975) and National Center for Advancing Translational Sciences (UL1-TR000445). We are grateful to Eugene E. Kim (New York University Medical Center [NYUMC]) for experimental advice; Thomas Jessell for providing the Etv1 mutant mouse line; Cindy Loomis and Mark Alu of the NYUMC Histopathology Core; Michael Gregory and Keith Kobylarz of the NYUMC Cytometry and Cell Sorting Core; and Adriana Heguy, Aristotelis Tsirigos, Steven Shen, and Betul Akgol Oksuz of the NYUMC Genome Technology Center, supported in part by the NYU Clinical and Translational Science Award UL1TR000038 from the National Center for Advancing Translational Sciences, NIH, and the Cancer Center Support Grant, P30CA016087, at the Laura and Isaac Perlmutter Cancer Center.

Address correspondence to: David S. Park, Leon H. Charney Division of Cardiology, New York University School of Medicine, 522 First Avenue, Smilow 803, New York, New York 10016, USA. Phone: 212.263.4131; E-mail: david.park@nyumc.org. Or to: Glenn I. Fishman, Leon H Charney Division of Cardiology, New York University School of Medicine, 522 First Avenue, Smilow 801, New York, New York 10016, USA. Phone: 212.263.3967; E-mail: glenn.fishman@nyumc.org.
1. Abraham WT, Hayes DL. Cardiac resynchronization therapy for heart failure. Circulation. 2003;108(21):2596-2603.

2. Remme CA, et al. The cardiac sodium channel displays differential distribution in the conduction system and transmural heterogeneity in the murine ventricular myocardium. Basic Res Cardiol. 2009;104(5):511-522.

3. Delorme B, et al. Developmental regulation of connexin 40 gene expression in mouse heart correlates with the differentiation of the conduction system. Dev Dyn. 1995;204(4):358-371.

4. Li GR, Lau CP, Shrier A. Heterogeneity of sodium current in atrial vs epicardial ventricular myocytes of adult guinea pig hearts. JMol Cell Cardiol. 2002;34(9):1185-1194.

5. Vaidyanathan $\mathrm{R}$, et al. The ionic bases of the action potential in isolated mouse cardiac Pur- kinje cell. Heart Rhythm. 2013;10(1):80-87.

6. Bagwe S, Berenfeld O, Vaidya D, Morley GE, Jalife J. Altered right atrial excitation and propagation in connexin 40 knockout mice. Circulation. 2005;112(15):2245-2253.

7. Tamaddon HS, Vaidya D, Simon AM, Paul DL, Jalife J, Morley GE. High-resolution optical mapping of the right bundle branch in connexin40 knockout mice reveals slow conduction 
in the specialized conduction system. Circ Res. 2000;87(10):929-936.

8. Linhares VL, et al. Transcriptional regulation of the murine Connexin 40 promoter by cardiac factors Nkx2-5, GATA4, and Tbx5. Cardiovasc Res. 2004;64(3):402-411.

9. Harris BS, et al. Differentiation of cardiac Purkinje fibers requires precise spatiotemporal regulation of Nkx2-5 expression. Dev Dyn. 2006;235(1):38-49.

10. Moskowitz IP, et al. A molecular pathway including Id 2, Tbx5, and Nkx2-5 required for cardiac conduction system development. Cell. 2007;129(7):1365-1376.

11. Basson CT, et al. Mutations in human TBX5 [corrected] cause limb and cardiac malformation in Holt-Oram syndrome. Nat Genet. 1997;15(1):30-35.

12. Benson DW, et al. Mutations in the cardiac transcription factor NKX2.5 affect diverse cardiac developmental pathways. J Clin Invest. 1999;104(11):1567-1573.

13. Li QY, et al. Holt-Oram syndrome is caused by mutations in TBX5, a member of the Brachyury (T) gene family. Nat Genet. 1997;15(1):21-29.

14. Schott JJ, et al. Congenital heart disease caused by mutations in the transcription factor NKX2-5. Science. 1998;281(5373):108-111.

15. Gutierrez-Roelens I, et al. A novel CSX/NKX2-5 mutation causes autosomal-dominant AV block: are atrial fibrillation and syncopes part of the phenotype? Eur J Hum Genet. 2006;14(12):1313-1316.

16. Moskowitz IP, et al. The T-Box transcription factor $\mathrm{Tbx} 5$ is required for the patterning and maturation of the murine cardiac conduction system. Development. 2004;131(16):4107-4116.

17. Pashmforoush M, et al. Nkx2-5 pathways and congenital heart disease; loss of ventricular myocyte lineage specification leads to progressive cardiomyopathy and complete heart block. Cell. 2004;117(3):373-386.

18. Jay PY, et al. Nkx2-5 mutation causes anatomic hypoplasia of the cardiac conduction system. J Clin Invest. 2004;113(8):1130-1137.

19. Briggs LE, et al. Perinatal loss of Nkx2-5 results in rapid conduction and contraction defects. Circ Res. 2008;103(6):580-590.

20. Rentschler S, et al. Neuregulin-1 promotes formation of the murine cardiac conduction system. Proc Natl Acad Sci US A. 2002;99(16):10464-10469.

21. Rentschler S, et al. Visualization and functional characterization of the developing murine cardiac conduction system. Development. 2001;128(10):1785-1792.

22. Denny JC, et al. PheWAS: demonstrating the feasibility of a phenome-wide scan to discover gene-disease associations. Bioinformatics. 2010;26(9):1205-1210.

23. Zhang SS, et al. Iroquois homeobox gene 3 establishes fast conduction in the cardiac HisPurkinje network. Proc Natl Acad Sci U S A. 2011;108(33):13576-13581.

24. Gassmann M, et al. Aberrant neural and cardiac development in mice lacking the ErbB4 neuregulin receptor. Nature. 1995;378(6555):390-394.

25. Lee KF, Simon H, Chen H, Bates B, Hung MC, Hauser C. Requirement for neuregulin receptor erbB2 in neural and cardiac development.
Nature. 1995;378(6555):394-398.

26. Baliga RR, et al. NRG-1-induced cardiomyocyte hypertrophy. Role of PI-3-kinase, p70(S6K), and MEK-MAPK-RSK. Am J Physiol.1999; 277(5 pt 2):H2026-H2O37.

27. Ren J, Bharti A, Raina D, Chen W, Ahmad R, Kufe D. MUC1 oncoprotein is targeted to mitochondria by heregulin-induced activation of c-Src and the molecular chaperone HSP90. Oncogene. 2006;25(1):20-31.

28. Liu J, Kern JA. Neuregulin-1 activates the JAK-STAT pathway and regulates lung epithelial cell proliferation. Am J Respir Cell Mol Biol. 2002;27(3):306-313.

29. Kim EE, et al. PCP4 regulates Purkinje cell excitability and cardiac rhythmicity. JClin Invest. 2014;124(11):5027-5036.

30. Pallante BA, et al. Contactin-2 expression in the cardiac Purkinje fiber network. Circ Arrhythm Electrophysiol. 2010;3(2):186-194.

31. Goel A, Janknecht R. Acetylation-mediated transcriptional activation of the ETS protein ER81 by p300, P/CAF, and HER2/Neu. Mol Cell Biol. 2003;23(17):6243-6254.

32. Baert JL, Monté D, Musgrove EA, Albagli O, Sutherland RL, de Launoit Y. Expression of the PEA3 group of ETS-related transcription factors in human breast-cancer cells. Int J Cancer. 1997;70(5):590-597.

33. Dowdy SC, Mariani A, Janknecht R. HER2/ Neu- and TAK1-mediated up-regulation of the transforming growth factor beta inhibitor Smad7 via the ETS protein ER81. J Biol Chem. 2003;278(45):44377-44384.

34. Bosc DG, Goueli BS, Janknecht R. HER2/Neumediated activation of the ETS transcription factor ER81 and its target gene MMP-1. Oncogene. 2001;20(43):6215-6224.

35. Wu J, Janknecht R. Regulation of the ETS transcription factor ER81 by the 90-kDa ribosomal S6 kinase 1 and protein kinase A. J Biol Chem. 2002;277(45):42669-42679.

36. Janknecht R. Regulation of the ER81 transcription factor and its coactivators by mitogen- and stress-activated protein kinase 1 (MSK1). Oncogene. 2003;22(5):746-755.

37. Dehorter N, Ciceri G, Bartolini G, Lim L, del Pino I, Marín O. Tuning of fast-spiking interneuron properties by an activity-dependent transcriptional switch. Science. 2015;349(6253):1216-1220.

38. Arber S, Ladle DR, Lin JH, Frank E, Jessell TM. ETS gene Er81 controls the formation of functional connections between group Ia sensory afferents and motor neurons. Cell. 2000;101(5):485-498.

39. Abe H, Okazawa M, Nakanishi S. Gene regulation via excitation and BDNF is mediated by induction and phosphorylation of the Etv1 transcription factor in cerebellar granule cells. Proc Natl Acad Sci U S A. 2012;109(22):8734-8739.

40. de Launoit Y, et al. The PEA3 group of ETS-related transcription factors. Role in breast cancer metastasis. Adv Exp Med Biol. 2000;480:107-116.

41. Sysa-Shah P, et al. Bidirectional cross-regulation between ErbB2 and $\beta$-adrenergic signalling pathways. Cardiovasc Res. 2016;109(3):358-373.

42. Akhtar S, Yousif MH, Chandrasekhar B, Benter IF. Activation of EGFR/ERBB2 via pathways involving ERK1/2, P38 MAPK, AKT and FOXO enhances recovery of diabetic hearts from ischemia-reperfusion injury. PLoS One. 2012;7(6):e39066.

43. Hattori F, et al. Nongenetic method for purifying stem cell-derived cardiomyocytes. Nat Methods. 2010;7(1):61-66.

44. Meysen S, et al. Nkx2.5 cell-autonomous gene function is required for the postnatal formation of the peripheral ventricular conduction system. Dev Biol. 2007;303(2):740-753.

45. Risebro CA, et al. Epistatic rescue of Nkx2.5 adult cardiac conduction disease phenotypes by prospero-related homeobox protein 1 and HDAC3. Circ Res. 2012;111(2):e19-e31.

46. Denny JC, et al. Systematic comparison of phenome-wide association study of electronic medical record data and genome-wide association study data. Nat Biotechnol. 2013;31(12):1102-1110.

47. Chi P, et al. ETV1 is a lineage survival factor that cooperates with KIT in gastrointestinal stromal tumours. Nature. 2010;467(7317):849-853.

48. Flames N, Hobert O. Gene regulatory logic of dopamine neuron differentiation. Nature. 2009;458(7240):885-889.

49. Li KX, et al. Neuregulin 1 regulates excitability of fast-spiking neurons through Kv1.1 and acts in epilepsy. Nat Neurosci. 2012;15(2):267-273.

50. Monté D, Coutte L, Baert JL, Angeli I, Stéhelin D, de Launoit Y. Molecular characterization of the ets-related human transcription factor ER81. Oncogene. 1995;11(4):771-779.

51. Brown TA, McKnight SL. Specificities of proteinprotein and protein-DNA interaction of GABP $\alpha$ and two newly defined ets-related proteins. Genes Dev. 1992;6(12B):2502-2512.

52. Zimmer C, et al. Role of Fgf8 signalling in the specification of rostral Cajal-Retzius cells. Development. 2010;137(2):293-302.

53. Chen Y, Hollemann T, Grunz H, Pieler T. Characterization of the Ets-type protein ER81 in Xenopus embryos. Mech Dev. 1999;80(1):67-76.

54. Shepherd TG, Kockeritz L, Szrajber MR, Muller WJ, Hassell JA. The pea3 subfamily ets genes are required for HER2/Neu-mediated mammary oncogenesis. Curr Biol. 2001;11(22):1739-1748.

55. Bosc DG, Janknecht R. Regulation of Her2/neu promoter activity by the ETS transcription factor, ER81. J Cell Biochem. 2002;86(1):174-183.

56. Lai D, et al. Neuregulin 1 sustains the gene regulatory network in both trabecular and nontrabecular myocardium. Circ Res. 2010;107(6):715-727.

57. Chen M, Lin YQ, Xie SL, Wang JF. Mitogenactivated protein kinase in endothelin-1-induced cardiac differentiation of mouse embryonic stem cells. J Cell Biochem. 2010;111(6):1619-1628.

58. Kim HS, Cho JW, Hidaka K, Morisaki T. Activation of MEK-ERK by heregulin-beta1 promotes the development of cardiomyocytes derived from ES cells. Biochem Biophys Res Commun. 2007;361(3):732-738.

59. Zhu WZ, Xie Y, Moyes KW, Gold JD, Askari B, Laflamme MA. Neuregulin/ErbB signaling regulates cardiac subtype specification in differentiating human embryonic stem cells. Circ Res. 2010;107(6):776-786.

60. Furtado MB, et al. A novel conditional mouse model for Nkx2-5 reveals transcriptional regu- 
lation of cardiac ion channels. Differentiation. 2016;91(1-3):29-41.

61. Hakuno D, Takahashi T, Lammerding J, Lee RT. Focal adhesion kinase signaling regulates cardiogenesis of embryonic stem cells. J Biol Chem. 2005;280(47):39534-39544.

62. Paulis L, et al. Activation of Sonic hedgehog signaling in ventricular cardiomyocytes exerts cardioprotection against ischemia reperfusion injuries. Sci Rep. 2015;5:7983.

63. Chen Z, et al. Endothelin-1 downregulates Mas receptor expression in human cardiomyocytes. Mol Med Rep. 2013;8(3):871-876.

64. Yue TL, et al. Inhibition of extracellular signal-regulated kinase enhances ischemia/ reoxygenation-induced apoptosis in cultured cardiac myocytes and exaggerates reperfusion injury in isolated perfused heart. Circ Res. 2000;86(6):692-699.

65. Gu A, Jie Y, Sun L, Zhao S, E M, You Q. RhNRG-1 protects the myocardium against irradiationinduced damage via the ErbB2-ERK-SIRT1 signaling pathway. PLoS One. 2015;10(9):e0137337.

66. Kesherwani V, Agrawal SK. Upregulation of RyR2 in hypoxic/reperfusion injury. J Neurotrauma. 2012;29(6):1255-1265.

67. $\mathrm{Li} \mathrm{J}$, et al. Anchored p90 ribosomal S6 kinase 3 is required for cardiac myocyte hypertrophy. Circ Res. 2013;112(1):128-139.

68. Li T, Liu Z, Hu X, Ma K, Zhou C. Involvement of ERK-RSK cascade in phenylephrine-induced phosphorylation of GATA4. Biochim Biophys Acta. 2012;1823(2):582-592.

69. Trogan E, Choudhury RP, Dansky HM, Rong JX, Breslow JL, Fisher EA. Laser capture microdissection analysis of gene expression in macrophages from atherosclerotic lesions of apolipoprotein E-deficient mice. Proc Natl Acad Sci U S A. 2002;99(4):2234-2239.

70. Kang G, et al. Purkinje cells from RyR2 mutant mice are highly arrhythmogenic but responsive to targeted therapy. Circ Res. 2010;107(4):512-519.

71. Bishopric NH, Kedes L. Adrenergic regulation of the skeletal alpha-actin gene promoter during myocardial cell hypertrophy. Proc Natl Acad Sci U S A. 1991;88(6):2132-2136.

72. Gutstein DE, Danik SB, Sereysky JB, Morley GE, Fishman GI. Subdiaphragmatic murine electrophysiological studies: sequential determination of ventricular refractoriness and arrhythmia induction. Am J Physiol Heart Circ Physiol. 2003;285(3):H1091-H1096.

73. Roden DM, et al. Development of a largescale de-identified DNA biobank to enable personalized medicine. Clin Pharmacol Ther. 2008;84(3):362-369.

74. Carroll RJ, Bastarache L, Denny JC. R PheWAS: data analysis and plotting tools for phenomewide association studies in the R environment. Bioinformatics. 2014;30(16):2375-2376. 